\title{
The VLT/NaCo large program to probe the occurrence of exoplanets and brown dwarfs at wide orbits ${ }^{\star} \star \star$
}

\section{Survey description, results, and performances}

\author{
G. Chauvin ${ }^{1}$, A. Vigan ${ }^{2}$, M. Bonnefoy ${ }^{3}$, S. Desidera ${ }^{4}$, M. Bonavita ${ }^{4}$, D. Mesa $^{4}$, A. Boccaletti ${ }^{5}$, E. Buenzli $^{3}$, \\ J. Carson ${ }^{6,3}$, P. Delorme ${ }^{1}$, J. Hagelberg 7 , G. Montagnier ${ }^{2}$, C. Mordasini ${ }^{3}$, S. P. Quanz ${ }^{8}$, D. Segransan ${ }^{7}$, C. Thalmann ${ }^{8}$, \\ J.-L. Beuzit ${ }^{1}$, B. Biller ${ }^{3}$, E. Covino ${ }^{9}$, M. Feldt ${ }^{3}$, J. Girard ${ }^{10}$, R. Gratton ${ }^{4}$, T. Henning ${ }^{3}$, M. Kasper ${ }^{11}$, A.-M. Lagrange ${ }^{1}$, \\ S. Messina ${ }^{12}$, M. Meyer ${ }^{8}$, D. Mouillet ${ }^{1}$, C. Moutou ${ }^{2}$, M. Reggiani ${ }^{8}$, J. E. Schlieder ${ }^{3}$, and A. Zurlo ${ }^{2}$ \\ ${ }^{1}$ UJF-Grenoble1/CNRS-INSU, Institut de Planétologie et d'Astrophysique de Grenoble UMR 5274, 38041 Grenoble, France \\ e-mail: Gael.Chauvin@obs.ujf-grenoble.fr \\ 2 Aix-Marseille Université, CNRS, LAM (Laboratoire d'Astrophysique de Marseille) UMR 7326, 13388 Marseille, France \\ 3 Max-Planck Institute for Astronomy, Königstuhl 17, 69117 Heidelberg, Germany \\ 4 INAF - Osservatorio Astronomico di Padova, Vicolo dell Osservatorio 5, 35122 Padova, Italy \\ 5 LESIA, Observatoire de Paris Meudon, 5 Pl. J. Janssen, 92195 Meudon, France \\ ${ }^{6}$ Department of Physics \& Astronomy, College of Charleston, 58 Coming Street, Charleston, SC 29424, USA \\ 7 Geneva Observatory, University of Geneva, Chemin des Mailettes 51, 1290 Versoix, Switzerland \\ ${ }^{8}$ Institute for Astronomy, ETH Zurich, Wolfgang-Pauli-Strasse 27, 8093 Zurich, Switzerland \\ 9 INAF Osservatorio Astronomico di Capodimonte via Moiarello 16, 80131 Napoli, Italy \\ 10 European Southern Observatory, Casilla 19001, Santiago 19, Chile \\ 11 European Southern Observatory, Karl Schwarzschild St, 2, 85748 Garching, Germany \\ 12 INAF - Catania Astrophysical Observatory, via S. So a 78, 95123 Catania, Italy
}

Received 3 February 2014 / Accepted 9 April 2014

\section{ABSTRACT}

\begin{abstract}
Context. Young, nearby stars are ideal targets for direct imaging searches for giant planets and brown dwarf companions. After the first-imaged planet discoveries, vast efforts have been devoted to the statistical analysis of the occurence and orbital distributions of giant planets and brown dwarf companions at wide ( $\geq 5-6$ AU) orbits.

Aims. In anticipation of the VLT/SPHERE planet-imager, guaranteed-time programs, we have conducted a preparatory survey of 86 stars between 2009 and 2013 to identify new faint comoving companions to ultimately analyze the occurence of giant planets and brown dwarf companions at wide (10-2000 AU) orbits around young, solar-type stars.

Methods. We used NaCo at VLT to explore the occurrence rate of giant planets and brown dwarfs between typically 0.1 and $8^{\prime \prime}$. Diffraction-limited observations in $H$-band combined with angular differential imaging enabled us to reach primary star-companion brightness ratios as small as $10^{-6}$ at $1.5^{\prime \prime}$. Repeated observations at several epochs enabled us to discriminate comoving companions from background objects.

Results. During our survey, twelve systems were resolved as new binaries, including the discovery of a new white dwarf companion to the star HD 8049. Around 34 stars, at least one companion candidate was detected in the observed field of view. More than 400 faint sources were detected; $90 \%$ of them were in four crowded fields. With the exception of HD 8049 B, we did not identify any new comoving companions. The survey also led to spatially resolved images of the thin debris disk around HD 61005 that have been published earlier. Finally, considering the survey detection limits, we derive a preliminary upper limit on the frequency of giant planets for the semi-major axes of $[10,2000]$ AU: typically less than $15 \%$ between 100 and 500 AU and less than $10 \%$ between 50 and 500 AU for exoplanets that are more massive than $5 M_{\text {Jup }}$ and $10 M_{\text {Jup }}$ respectively, if we consider a uniform input distribution and a confidence level of $95 \%$

Conclusions. The results from this survey agree with earlier programs emphasizing that massive, gas giant companions on wide orbits around solar-type stars are rare. These results will be part of a broader analysis of a total of $\sim 210$ young, solar-type stars to bring further statistical constraints for theoretical models of planetary formation and evolution.
\end{abstract}

Key words. instrumentation: adaptive optics - instrumentation: high angular resolution - methods: observational - brown dwarfs techniques: image processing - planetary systems

\footnotetext{
* Based on observations collected at the European Southern Observatory, Chile (ESO Large Program 184.C-0157 and Open Time 089.C-0137A and 090.C-0252A).

$\star \star$ Tables 2 and 6 are available in electronic form at http://www . aanda.org
}

\section{Introduction}

Our understanding of the origin and evolution of extrasolar planets (EPs) has drastically transformed in the last decade. Current theories favor the formation of planets within a protoplanetary disk by the accretion of solids, which build up a 10 to $15 M_{\oplus}$ 
core followed by rapid agglomeration of gas (Pollack et al. 1996; Alibert et al. 2004), or by gravitational instability of the gas (Boss 1997; Stamatellos \& Withworth 2008; Vorobyov 2013). Whereas physical conditions and timescales favor core accretion in the inner disk $(\leq 10 \mathrm{AU})$, gravitational instability could be the main mechanism to form massive gaseous giants at wider separations ( $\geq 10 \mathrm{AU})$ in the earliest phase of the disk's lifetime (Boley 2009). The planets could migrate either inward, toward, or outward from the star by disk-planet interactions (Kley \& Nelson 2012 and reference therein) or during planet-planet interactions (Naoz et al. 2011; Dawson \& Murray-Clay 2013), which alter the original semi-major axis distribution. A wide range of potential planet masses, sizes, locations, and compositions results from this flurry of formation and evolution possibilities. A major goal for exoplanetary science in the next decade is a better understanding of these mechanisms. In this context, the role of observations is crucial in providing constraints that will help to model the diversity of exoplanetary properties. The main observables are the occurrence of EPs, the physical properties and orbital characteristics (composition, mass, radius, luminosity, distribution of mass, period, and eccentricity) but also the properties of the planetary hosts (mass, age, metallicity, lithium abundance, or multiplicity).

Brown dwarfs (BDs) were originally proposed as a distinguishable class of astrophysical objects with intermediate masses between stars and planets. Recent large infrared surveys and high contrast observations have unambiguously revealed the existence of planetary mass objects, which are isolated in the field (Zapatero-Osorio et al. 2000; Liu et al. 2013; Joergens et al. 2013) or wide companions to stars (Chauvin et al. 2005a). Their existence confirms that the formation mechanisms proposed to form stars (gravo-turbulent fragmentation, disk fragmentation, accretion-ejection or photo-erosion; see Whitworth et al. 2007; Luhman 2012 for reviews) can actually form objects down to the planetary mass regime. The details of contraction and subsequent evolution of the cores remain critical and are still under considerable debate. Episodic accretion processes can affect their physical properties (Baraffe et al. 2009). It is now undeniable that the stellar and planetary formation mechanisms overlap in the substellar regime. They can both lead to the formation of planetary mass objects, including companions to stars and BDs. Fossil traces of the formation processes should be revealed by different physical features (presence of core, composition of the atmosphere, system architecture...). Distinct statistical properties such as the occurrence, the mass, separation and eccentricity distributions, should help to identify the dominant mechanism to form substellar companions.

The main statistical constraints on exoplanets originally came from the radial velocity (RV) technique. More than 800 EPs have been now confirmed, which feature a broad range of physical (mass) and orbital $(\mathrm{P}, e)$ characteristics around different stellar hosts (Howard et al. 2010; Mayor et al. 2011; Wright et al. 2012; Bonfils et al. 2013). The strong bimodal aspect of the secondary-mass distribution to solar-type primaries has generally been considered the most obvious evidence of different formation mechanisms for stellar and planetary systems. The period distribution of giant exoplanets is basically made of two main features: a peak around 3 days plus an increasing frequency as a function of period (Udry \& Santos 2007). The observed pile up of planets with periods around 3 days is believed to be the result of migration and final stopping mechanism. The rise of the number of planets with increasing distance from the parent star reaches up to a separation corresponding to the duration limit of most of the longest surveys (5-6 AU).
This extrapolation hints that a large population of yet undetected Jupiter-mass planets may exist beyond 5 AU, suggesting an ideal niche for the direct-imaging surveys. More recently, a plethora of transiting planetary candidates have been revealed by Kepler (more than 2300 candidates known today, Batalha et al. 2013), which probably corroborate how abundant telluric planets are and agrees with Doppler surveys in terms of occurrence at less than 0.25 AU (Howard et al. 2012).

Despite the success of the RV and transit techniques, the time spans explored limit the studies to the close ( $\leq 5-6$ AU) EPs. Within the coming years, direct imaging represents the only viable technique for probing the existence of EPs and BD companions at large ( $\geq 5-6$ AU) separations. This technique is also unique for the characterization of planetary atmospheres that are not strongly irradiated by the planetary host (Janson et al. 2010; Bowler et al. 2010; Barman et al. 2011a,b; Bonnefoy et al. 2010, 2013, 2014a,b; Konopacky et al. 2013). Young ( $\leq 500 \mathrm{Myr})$, nearby stars are very favorable targets for the direct detection of the lowest mass companions. Since the discovery of the TW Hydrae association (TWA) by Kastner et al. (1997) and Hoff et al. (1998), more than 300 young, nearby stars were identified. They are gathered in several groups (TWA, $\beta$ Pictoris, Tucana-Horologium, $\eta$ Cha, AB Dor, Columba, Carinae), sharing common kinematics and photometric and spectroscopic properties (see Zuckerman \& Song 2004; Torres et al. 2008). With typical contrast of 10-15 magnitudes for separations beyond 1.0-2.0" (50-100 AU for a star at $50 \mathrm{pc})$, planetary mass companions down to 1-2 Jupiter masses are potentially detectable by current imaging surveys that are very deep. The first planetary mass companions were detected at large distances $(\geq 100 \mathrm{AU})$ and/or with small mass ratio with their primaries, indicating a probable star-like or gravitational disk instability formation mechanism (Chauvin et al. 2005b; Lafrenière et al. 2008).

The breakthrough discoveries of closer and/or lighter planetary mass companions like Fomalhaut b $\left(<1 M_{\text {Jup }}\right.$ at $177 \mathrm{AU}$; Kalas et al. 2008, 2013), HR 8799 bcde (10, 10, 10 and $7 M_{\text {Jup }}$ at resp. 14, 24, 38, and $68 \mathrm{AU}$; Marois et al. 2008, 2010), $\beta$ Pictoris b ( $8 M_{\mathrm{Jup}}$ at $8 \mathrm{AU}$; Lagrange et al. 2009), or more recently $\kappa$ and b $\left(14_{-2}^{+25} M_{\text {Jup }}\right.$ at 55 AU; Carson et al. 2013; Bonnefoy et al. 2014b), HD 95086b (4-5 $M_{\text {Jup }}$ at $56 \mathrm{AU}$; Rameau et al. 2013a,b), and GJ 504 b (4+1 ${ }_{-1}^{+4.5} M_{\text {Jup }}$ at $43.5 \mathrm{AU}$; Kuzuhara et al. 2013) indicate that we are just initiating the characterization of the outer part of planetary systems between typicaly 5-100 AU. Vast efforts are now devoted to systematic searches of EPs in direct imaging with an increasing number of large scale surveys (see Table 1; nine new surveys published between 2012 and 2013). The number of targets surveyed and the detection performances will increase with the new generation of planet finders LMIRCam at LBT (Skrutskie et al. 2010), MagAO (Close et al. 2012), ScExAO at Subaru (Guyon et al. 2010), SPHERE at VLT (Beuzit et al. 2008), and GPI at Gemini (Macintosh et al. 2008) with the goal to provide better statistics on larger samples and a greater number of giant planets to be characterized. It should enable the testing of alternative mechanisms to the standard planetary formation theories of core accretion and gravitation instability such as pebble accretion (Lambrechts \& Johansen 2012; Morbidelli \& Nesvorny 2012) or tidal downsizing (Boley et al. 2010; Nayakshin 2010; Forgan \& Rice 2013) that are currently proposed to explain the existence of a population of giant planets at wide orbits. In the context of the VLT/SPHERE scientific preparation, we have conducted a large observing program (ESO: 184.C-0157) of 86 stars with $\mathrm{NaCo}$ 
G. Chauvin et al.: The VLT/NaCo large program to probe the occurrence of exoplanets in wide orbits. II.

Table 1. Deep imaging surveys of young $(<100 \mathrm{Myr})$ and intermediate-old to old $(0.1-5 \mathrm{Gyr})$, close $(<100 \mathrm{pc})$ stars that are dedicated to the search for planetary mass companions.

\begin{tabular}{|c|c|c|c|c|c|c|c|c|}
\hline Reference & Telescope & Instr. & Mode & Filter & $\begin{array}{l}\text { FoV } \\
\left({ }^{\prime \prime} x^{\prime \prime}\right)\end{array}$ & $\#$ & $\mathrm{SpT}$ & $\begin{array}{l}\text { Age } \\
\text { (Myr) }\end{array}$ \\
\hline Chauvin et al. (2003) & ESO3.6m & ADONIS & Cor-I & $H, K$ & $13 \times 13$ & 29 & GKM & $\$ 50$ \\
\hline \multirow[t]{2}{*}{ Neuhäuser et al. (2003) } & NTT & Sharp & Sat-I & $K$ & $11 \times 11$ & 23 & AFGKM & $\$ 50$ \\
\hline & NTT & Sofi & Sat-I & $H$ & $13 \times 13$ & 10 & AFGKM & $\lesssim 50$ \\
\hline Lowrance et al. (2005) & HST & NICMOS & Cor-I & $H$ & $19 \times 19$ & 45 & AFGKM & $10-600$ \\
\hline Masciadri et al. (2005) & VLT & $\mathrm{NaCo}$ & Sat-I & $H, K$ & $14 \times 14$ & 28 & KM & $\lesssim 200$ \\
\hline \multirow[t]{2}{*}{ Biller et al. (2007) } & VLT & $\mathrm{NaCo}$ & SDI & $H$ & $5 \times 5$ & 45 & GKM & $\$ 300$ \\
\hline & MMT & & SDI & $H$ & $5 \times 5$ & - & - & - \\
\hline Kasper et al. (2007) & VLT & $\mathrm{NaCo}$ & Sat-I & $L^{\prime}$ & $28 \times 28$ & 22 & GKM & $\lesssim 50$ \\
\hline Lafrenière et al. (2007) & Gemini-N & NIRI & ADI & $H$ & $22 \times 22$ & 85 & & $10-5000$ \\
\hline Apai et al. $(2008)^{a}$ & VLT & $\mathrm{NaCo}$ & SDI & $H$ & $3 \times 3$ & 8 & FG & $12-500$ \\
\hline Chauvin et al. (2010) & VLT & $\mathrm{NaCo}$ & Cor-I & $H, K$ & $28 \times 28$ & 88 & BAFGKM & $\lesssim 100$ \\
\hline Heinze et al. $(2010 a, b)$ & MMT & Clio & ADI & $L^{\prime}, M$ & $15.5 \times 12.4$ & 54 & FGK & $100-5000$ \\
\hline Janson et al. (2011) & Gemini-N & NIRI & ADI & $H, K$ & $22 \times 22$ & 15 & BA & $20-700$ \\
\hline \multirow[t]{2}{*}{ Vigan et al. (2012) } & Gemini-N & NIRI & ADI & $H, K$ & $22 \times 22$ & 42 & $\mathrm{AF}$ & $10-400$ \\
\hline & VLT & $\mathrm{NaCo}$ & ADI & $H, K$ & $14 \times 14$ & - & - & - \\
\hline Delorme et al. (2012) & VLT & $\mathrm{NaCo}$ & $\mathrm{ADI}$ & $L^{\prime}$ & $28 \times 28$ & 16 & M & $\lesssim 200$ \\
\hline Rameau et al. (2013c) & VLT & $\mathrm{NaCo}$ & ADI & $L^{\prime}$ & $28 \times 28$ & 59 & $\mathrm{AF}$ & $\lessgtr 200$ \\
\hline Yamamoto et al. (2013) & Subaru & HiCIAO & ADI & $H, K$ & $20 \times 20$ & 20 & FG & $125 \pm 8$ \\
\hline Biller et al. (2013) & Gemini-S & NICI & Cor-ASDI & $H$ & $18 \times 18$ & 80 & BAFGKM & $\lesssim 200$ \\
\hline Brandt et al. (2013) & Subaru & HiCIAO & ADI & $H$ & $20 \times 20$ & 63 & AFGKM & $\lesssim 500$ \\
\hline Nielsen et al. (2013) & Gemini-S & NICI & Cor-ASDI & $H$ & $18 \times 18$ & 70 & BA & $50-500$ \\
\hline Wahhaj et al. (2013) ${ }^{a}$ & Gemini-S & NICI & Cor-ASDI & $H$ & $18 \times 18$ & 57 & AFGKM & $\sim 100$ \\
\hline Janson et al. (2013) ${ }^{a}$ & Subaru & HiCIAO & ADI & $H$ & $20 \times 20$ & 50 & AFGKM & $\lesssim 1000$ \\
\hline
\end{tabular}

Notes. We have indicated the telescope, the instrument, the imaging mode (Cor-I: coronagraphic imaging; Sat-I; saturated imaging; I: imaging; SDI: simultaneous differential imaging; ADI: angular differential imaging; ASDI: angular and spectral differential imaging), the filters, the field of view (FoV), the number of stars observed (\#), their spectral types (SpT), and ages (Age). ${ }^{(a)}$ Surveys dedicated to planets around debris disk stars.

(hereafter the NaCo-LP). Combined with stars already observed in direct imaging, it represents a total of more than $\sim 210$ stars for studying the occurrence rate of giant planets and brown dwarf companions at wide (10-2000 AU) orbits. This complete analysis is detailed in a series of four papers: a description of the complete sample (Desidera et al. 2015), the NaCo-LP survey (this paper), the statistical analysis of the giant planet population (Vigan et al., in prep.), and that of the brown dwarf companion population (Reggiani et al., in prep.). We therefore report here the results of the NaCo-LP carried out between 2009 and 2013. In Sect. 2, we describe the target sample selection. In Sect. 3, we describe the details of the observing setup. In Sect. 4, the data reduction strategy and analysis are reported with the results in Sect. 5. Finally, a preliminary statistical analysis of the observed sample is presented in Sect. 6 and our main conclusions in Sect. 7.

\section{Target properties}

Based on a complete compilation of young, nearby stars that have been recently identified in young co-moving groups and from systematic spectroscopic surveys, we have selected a sample of stars according to their declination $\left(\delta \leq 25^{\circ}\right)$, their age ( $\$ 200 \mathrm{Myr}$ ), their distance $(d \lesssim 100 \mathrm{pc})$, and their $R$-band brightness $(R \leq 9.5)$. In addition, none of these stars had been observed in a high-contrast imaging survey before. Great care has been taken in the age selection criteria based on different youth diagnostics (isochrones, lithium abundance, $\mathrm{H} \alpha$ emission, X-ray activity, stellar rotation, chromospheric activity, and kinematics). Close visual (0.1-6.0") and spectroscopic binaries were rejected as they degrade the VLT/NaCo detection performances and bias the astrophysical interpretation. Among this sample, 86 stars were finally observed during the large program. The main target properties (spectral type, distance, age, $H$-band magnitude, galactic latitude, and proper motion) are reported in Table 2. They are also shown in Fig. 1 with the properties of the complete statistical sample used by Vigan et al. (in prep.) and Reggiani et al. (in prep.). A complete characterization of the $\mathrm{NaCo}-\mathrm{LP}$ observed sample and the archive sample, particularly, with regard to the age and distance determination, is determined by Desidera et al. (2015). As can be seen from Fig. 1, the core of the NaCoLP observed sample is mainly composed of close young (10200 Myr) solar-type FGK stars.

\section{Observations: telescope and instrument}

We used the NaCo high contrast Adaptive Optics (AO) imager of the VLT-UT4. The NaCo instrument is equipped with the NAOS AO system (Rousset et al. 2002), and the near-infrared imaging camera CONICA (Lenzen et al. 2002). The observations were obtained during various observing runs spread between the end of 2009 and 2013 in visitor and service (queue-observing) modes. The summary of the observing runs is reported in Table 3. The NaCo-LP represents a total of 16.5 observing nights, 10.5 nights obtained in visitor mode and 6 nights in service.

To achieve high contrasts, we used angular differential imaging (ADI) on pupil-stabilized mode of NaCo. A classical Lyot-coronagraph with a diameter of $0.7^{\prime \prime}$ was used during the first visitor run but then replaced by saturated imaging as the 



Fig. 1. Histrograms summarizing the main properties of NaCo-LP observed sample (dark blue) and of the final NaCo-LP statistical sample of $\sim 210$ stars (light blue) used by Vigan et al. (in prep.) and Reggiani et al. (in prep.): spectral type, age, distance, $H$-band magnitude, proper motion amplitude, and galactic latitude.

Table 3. Observing campaigns.

\begin{tabular}{llllll}
\hline \hline ESO Program & Mode & $\begin{array}{l}\text { St. Night } \\
(\text { UT-date) }\end{array}$ & $\begin{array}{l}\text { Night } \\
(\mathrm{Nb})\end{array}$ & $\begin{array}{l}\text { Loss } \\
(\%)\end{array}$ & $\begin{array}{l}\text { Visit } \\
(\mathrm{Nb})\end{array}$ \\
\hline 184.C-0157A & LP-Vis & $2009-11-21$ & 3 & 20 & 23 \\
184.C-0157E & LP-Vis & $2010-02-16$ & 3 & 0 & 27 \\
184.C-0157B & LP-Vis & $2010-06-14$ & 2.5 & 70 & 11 \\
184.C-0157F & LP-Vis & $2010-07-29$ & 2 & 33 & 18 \\
184.C-0157C & LP-Ser & - & 1.5 & 0 & 15 \\
184.C-0157D & LP-Ser & - & 3.3 & 0 & 33 \\
089.C-0137A & OT-Ser & - & 0.7 & 0 & 6 \\
090.C-0252A & OT-Ser & - & 0.5 & 0 & 4 \\
\hline Total & - & - & 16.5 & & 137 \\
\hline
\end{tabular}

Notes. The table includes the ESO-program number, the observing mode (LP for Large-Program, OT for Open-Time, Vis for Visitor run, and Ser for Service run), the starting night, and the number of nights, the observing loss (technical and weather), and the number of observing sequences, including single and multiple visits per target.

NaCo point spread function (PSF) was unexpectedly drifting with time owing to a technical problem with the instrument. For accurate astrometry, a single observing setup was used, corresponding to the combined use of the $H$-band filter with the S13 camera (13.25 mas/pix). The time of the observations were chosen to maximize the field rotation. Typical exposure times of $1-10 \mathrm{~s}$ were used to saturate the PSF core by a factor 100 (a few pixels in radius) to improve the dynamic range of our images. The NaCo detector cube mode was additionally used to register each individual frame to optimize the final image selection in post-processing. The typical observing sequence was composed of a total of 10-15 cubes of 10-120 frames, which has a total integration time of 35-40 min for an observing sequence of 1-1.5 hrs on target. The parallactic angle variations are reported in Fig. 2 with the airmass, coherent energy, coherent time, as measured by $\mathrm{NaCo}$, and the seeing, as measured by the DIMM seeing monitor at VLT. Non-saturated PSFs were acquired in ADI using a neutral density filter at the beginning of each observing sequence to monitor the image quality. They also served for the calibration of the relative photometric and astrometric measurements.

\section{Data reduction and analysis}

\subsection{Cosmetics and data processing}

Three independent pipelines were used to reduce and analyze the ADI data to optimize the PSF subtraction and the detection performances and to check the consistency of the results in terms of astrometry and photometry. These pipelines are described for the LAM-ADI pipeline by Vigan et al. (2012), the IPAG-ADI pipeline by Chauvin et al. (2012), and the PadovaADI pipeline by Esposito et al. (2013). Each pipeline processed the data in a similar way for the first cosmetic steps of flatfielding, bad- and hot-pixel removal, and sky subtraction. To determine the central star position for the frame recentring, a Moffat fitting of the non-saturated part of the stellar PSF wing (with a similar threshold) was used. Finally, an encircled energy criteria was considered for the rejection of open-loop and poorly-corrected frames for computing a final mastercube with the correspoding parallactic angle variation. The main differences between the pipelines mostly reside in the various ADI algorithms applied (cADI and sADI, see Marois et al. 2006; LOCI, see Lafrenière et al. 2007) and in the parameters setup. Consistent results within $0.1-0.2$ mag in photometry (candidate photometry and detection limits) and $0.2-0.3$ pixels in astrometry were found between the different pipelines for a series of targets used as test cases. Non-saturated PSFs were similarly reduced without PSF subtraction.

The results presented in this final analysis have been obtained with the LAM-ADI pipeline using LOCI with optimization regions of $N_{\mathrm{A}}=300 \times F W H M$ at less than $3^{\prime \prime}, N_{\mathrm{A}}=$ $3000 \times F W H M$ at more than $3^{\prime \prime}$, the radial to azimuthal width ratio $g=1$, the radial width $\Delta r=2 \times F W H M$, and a separation criteria of $0.75 \times F W H M$. The binning of the data was tuned to apply LOCI on a final mastercube taht is reduced to $\sim 350$ frames. An illustration of the final LOCI processed image of the young star TYC 7617-0549-1 (K0V, 76.4 pc and 30 Myr) is shown in Fig. 3. 
G. Chauvin et al.: The VLT/NaCo large program to probe the occurrence of exoplanets in wide orbits. II.
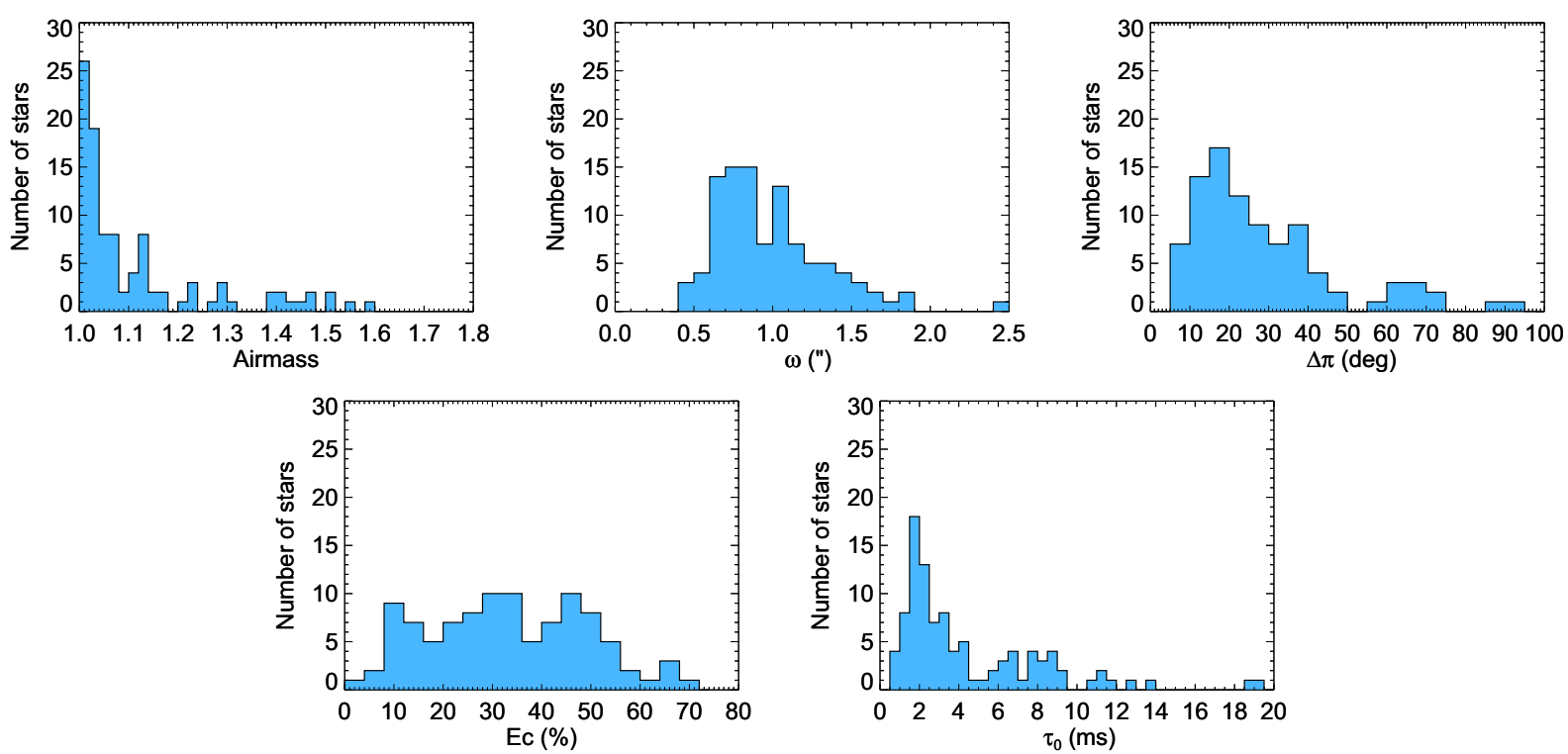

Fig. 2. Histograms summarizing the observing conditions of the NaCo-LP campaigns: airmass, DIMM seeing $(\omega)$, parallactic angle variation $(\Delta \pi)$, coherent energy $(\mathrm{Ec})$, and coherent time $\left(\tau_{0}\right)$.

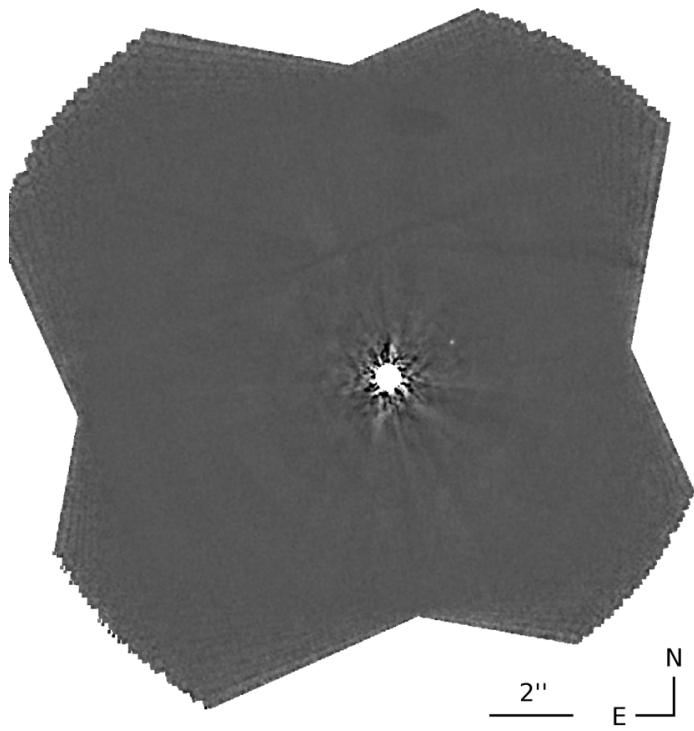

Fig. 3. VLT/NACO ADI observation in $H$-band of the young star TYC 7617-0549-1 (K0V, $76.4 \mathrm{pc}$ and $30 \mathrm{Myr})$. A faint ( $\Delta H=12.7 \mathrm{mag}$ ) candidate, resolved at $1.8^{\prime \prime}$, has been finally identified as a background contaminant (see Fig. 6).

\subsection{Relative astrometry and photometry}

The relative position and flux of all candidates was determined using Moffat fitting and aperture photometry corrected from the ADI flux loss. This first order analysis was sufficient for assessing the proper motion and nature of the candidate as described in Sect. 5.2. For the most interesting cases (like HD 8049), the injection of fake planets at the location of the candidate signal was done to properly take any local astrometric and photometric biases into account which induced by the ADI-processing described in Chauvin et al. (2012).

To finally calibrate the relative astrometric position of the detected candidates to the primary star, we used the $\theta_{1}$ Ori $C$ field observed with HST by McCaughrean \& Stauffer (1994; with the same set of stars TCC058, 057, 054, 034 and 026) as a primary
Table 4. Mean plate scale and true north orientation for each observing run of the NaCo-LP.

\begin{tabular}{llll}
\hline \hline UT Date & $\begin{array}{l}\text { Platescale } \\
(\mathrm{mas})\end{array}$ & $\begin{array}{l}\text { True north } \\
(\mathrm{deg})\end{array}$ & Calibrator \\
\hline $2009-11-23$ & $13.22 \pm 0.02$ & $-0.17 \pm 0.03$ & $\theta_{1}$ Ori C \\
$2010-02-15$ & $13.21 \pm 0.02$ & $-0.33 \pm 0.03$ & IDS1307 \\
$2010-02-18$ & $13.21 \pm 0.02$ & $-0.33 \pm 0.03$ & $\theta_{1}$ Ori C \\
$2010-06-16$ & $13.21 \pm 0.02$ & $-0.53 \pm 0.03$ & IDS1307 \\
$2010-07-30$ & $13.21 \pm 0.02$ & $-0.47 \pm 0.03$ & IDS1307 \\
$2010-12-30$ & $13.21 \pm 0.02$ & $-0.47 \pm 0.03$ & $\theta_{1}$ Ori C \\
$2011-01-30$ & $13.21 \pm 0.02$ & $-0.49 \pm 0.03$ & $\theta_{1}$ Ori C \\
$2011-05-11$ & $13.21 \pm 0.02$ & $-0.52 \pm 0.03$ & IDS1307 \\
$2011-07-02$ & $13.21 \pm 0.02$ & $-0.55 \pm 0.03$ & IDS1307 \\
$2012-01-02$ & $13.22 \pm 0.02$ & $-0.59 \pm 0.03$ & IDS1307 \\
$2012-01-02$ & $13.22 \pm 0.02$ & $-0.59 \pm 0.03$ & $\theta_{1}$ Ori C \\
\hline
\end{tabular}

calibrator. The astrometric binary IDS 13022N0107 (van Dessel $\&$ Sinachopoulos 1993) was then used as a secondary calibrator when the $\theta_{1}$ Ori $\mathrm{C}$ field was not observable and then recalibrated on the $\theta_{1}$ Ori $\mathrm{C}$ field when both were observable. Both fields were observed in standard field-stabilized mode and reduced (cosmetics, flat-fielding, bad and hot-pixel removal, sky subtraction, and recentring) using the Eclipse $e^{1}$ reduction software developed by Devillar (1997). Finally, for ADI data, the $\mathrm{NaCo}$ rotator offset at the start of each ADI sequence was also calibrated and taken into account as described by Chauvin et al. (2012). The results of the platescale and true north orientation determinations are given in Table 4.

The throughput of the NaCo neutral density filter was recalibrated on sky using two different datasets taken for the star TYC 9162-0698 during our February 2010 visitor run. Using aperture photometry on the data taken with and without the neutral density, we derived a transmission factor of $1.19 \pm 0.05 \%$ with the $H$-band filter. This result is consistent with the one derived by Bonnefoy et al. (2013) and was used to calibrate the candidate photometry and the detection limits using the nonsaturated sequence of the primary star with the neutral density filter as a photometric reference.

1 http://www.eso.org/projects/aot/eclipse/ 


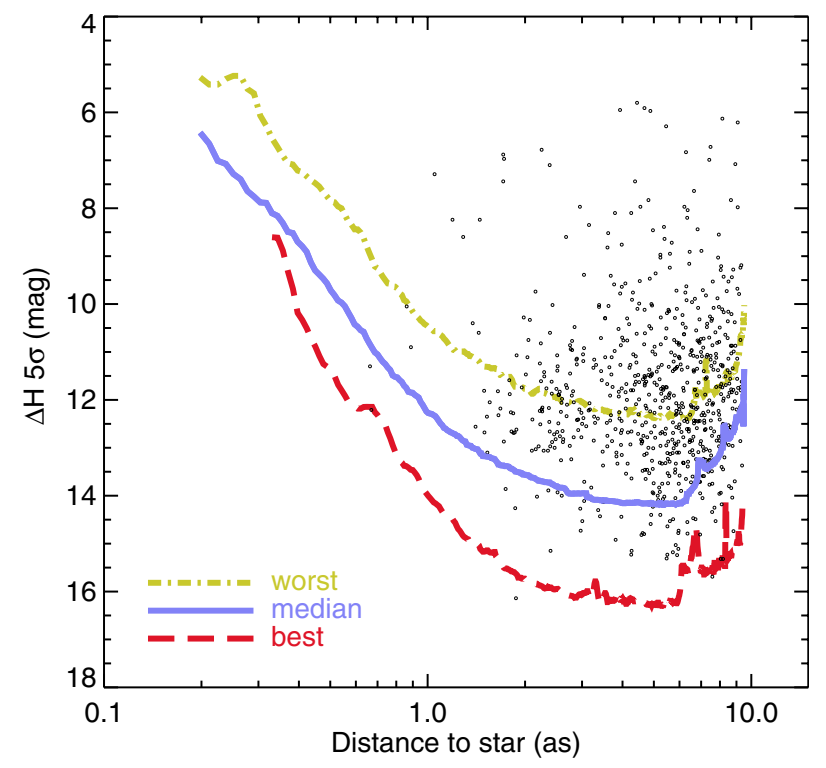

Fig. 4. VLT/NACO deep ADI $5 \sigma$ detection limits in $H$-band combined with the S13 camera. The worst, median, and best detection limits are shown with all the candidates detected. Separations of less than $0.1-0.2^{\prime \prime}$ are generally saturated.

\subsection{Detection limit determination}

A pixel-to-pixel noise map of each observation was estimated within a box of $5 \times 5$ pixels sliding from the star to the limit of the NACO field of view. To correct for the flux loss related to the ADI processing, fake planets were regularly injected for every 20 pixels in radius at 10 different position angles for separations smaller than $3^{\prime \prime}$. At more than $3^{\prime \prime}$, fake planets were injected for every 50 pixels at four different position angles. The final flux loss was computed with the azimuthal average of the flux losses of fake planets at the same radii. The final detection limits at $5 \sigma$ were then obtained using the pixel-to-pixel noise map divided by the flux loss and normalized by the relative calibration with the primary star (considering the different exposure times and the neutral density). The LOCI processing leads to residuals whose distribution closely resembles a Gaussian (Lafrenière et al. 2007); therefore, a $5 \sigma$ threshold is thus adequate for estimating detection performances. The best, worst and median detection limits of the survey are reported in Fig. 4.

\section{Results}

A total of 86 sources were observed. Sixteen stars were resolved as binaries, including HD 8049 with a newly discovered white dwarf companion. Ten binaries were simply observed in nonsaturated ADI imaging to directly derive their relative astrometry and photometry. Seventy-six stars were observed in saturated high-contrast ADI to search for faint substellar companions. In the following sub-sections, we describe the properties of the new stellar multiple systems, the status of the detected candidates in saturated ADI, the characteristics of the white dwarf companion around HD 8049, and finally the fine analysis of the thin debrisdisk around HD 61005.

\subsection{New stellar close multiple systems}

Despite our sample selection to reject close $\left(0.1-6.0^{\prime \prime}\right)$ binaries, 16 stars were resolved as multiple. Three systems were already
Table 5. Relative positions and $H$-band contrast of the new binaries resolved during the $\mathrm{NaCo}-\mathrm{LP}$.

\begin{tabular}{llll}
\hline \hline Name & $\begin{array}{l}\Delta \\
(\mathrm{mas})\end{array}$ & $\begin{array}{l}\text { PA } \\
(\mathrm{deg})\end{array}$ & $\begin{array}{l}\Delta H \\
(\mathrm{mag})\end{array}$ \\
\hline HIP 8038 & $437 \pm 7$ & $273.8 \pm 0.9$ & $2.5 \pm 0.2$ \\
HIP 80290 & $3340 \pm 4$ & $257.5 \pm 0.2$ & $1.9 \pm 0.2$ \\
HIP 94235 & $506 \pm 7$ & $150.6 \pm 0.8$ & $3.8 \pm 0.3$ \\
HIP 107684 & $326 \pm 7$ & $270.2 \pm 1.2$ & $2.8 \pm 0.3$ \\
HD 199058 & $471 \pm 7$ & $282.9 \pm 0.8$ & $2.5 \pm 0.2$ \\
TYC 0603-0461-1 & $74 \pm 14$ & $74.2 \pm 5.1$ & $0.1 \pm 0.4$ \\
TYC 8927-3620-1 & $87 \pm 14$ & $296.8 \pm 4.35$ & $0.5 \pm 0.4$ \\
TYC 8989-0583-1 $^{a}$ & $2584 \pm 8$ & $169.8 \pm 0.15$ & $2.7 \pm 0.2$ \\
TYC 9010-1272-1 & $262 \pm 8$ & $238.0 \pm 1.44$ & $1.0 \pm 0.3$ \\
TYC 9181-0466-1 & $1891 \pm 7$ & $123.4 \pm 0.2$ & $1.5 \pm 0.2$ \\
TYC 9231-1566-1 & $1975 \pm 7$ & $145.5 \pm 0.2$ & $3.0 \pm 0.2$ \\
\hline
\end{tabular}

Notes. Epochs of observation are reported in Table 6. ${ }^{(a)}$ Known binary separated by $15.0 \operatorname{arcsec}$ and $\Delta V=2.0$ mag. ${ }^{(b)}$ Third component resolved by 2 MASS at $\sim 4.8^{\prime \prime}$ and $\Delta K=0.7 \mathrm{mag}$.

known, HIP 108422 AB (Chauvin et al. 2003), TYC 7835-2569$1 \mathrm{AB}$ (Brandner et al. 1996), and TYC 6786-0811-1 (Köhler et al. 2000), and went through our sample selection process by mistake. The system TYC $8484-1507-1$ is actually also a known $\sim 8.6^{\prime \prime}$ binary that was resolved by $2 \mathrm{MASS}$, which is not rejected during our sample selection but resolved in the $\mathrm{NaCo}$ FoV, despite its large separation. Then, in the case of HD 8049, the faint comoving companion turned out to be a white dwarf. Its characteristics are briefly described in Sect. 5.3. At the end, a total of eleven new close multiple systems were resolved. All of them were observed in non-saturated ADI to derive their position and $H$-band photometry relative to the primary star (see Table 5). The visual binaries HIP 108422 AB, TYC 78352569-1 AB and TYC 6786-0811-1 are confirmed as physically bound. Deep ADI observations were obtained in addition to six binaries (TYC 0603-0461-1, TYC 7835-2569-1, HD 8049, TYC 8927-3620-1, HIP 80290, and TYC 8989-0583-1).

\subsection{Companion candidates}

Among the 76 stars observed in ADI, one companion candidate or more were detected for 43 targets (see Table 2). More than 700 candidates were detected with $90 \%$ of them in six very crowded field (see Fig. 4). The galactic contamination rate, predicted by the Besançon galactic population model (Robin et al. 2003) for the NaCo-LP fields and at least one background source, is equal to $51 \%$, which reasonably agrees with $56 \%$ (43 systems with at least one candidate for the 76 observed). The model uses the NaCo field of view as input with the typical magnitude limit of the NaCo-LP $\left(H_{\text {lim }}=21 \mathrm{mag}\right)$ survey, and the galactic coordinates of all targets. The repartition of these galactic contaminants is given in Fig. 5. Solar-system and extragalactic contaminants are expected to be significantly less frequent. Moreover, solar system contaminants smear during a $1 \mathrm{~h}$ observing sequence, and extra-galactic contaminants are mainly extended background galaxies resolved by $\mathrm{NaCo}$. The most important population of contaminants that can mimic the apparent flux of the giant planet or brown dwarf companions bound to the star are $\mathrm{M}$ dwarfs with typical $H=20-22 \mathrm{mag}$ apparent magnitudes.

To identify their nature, we relied on the follow-up observations at additional epochs to distinguish comoving companions from stationary background stars. The candidates were ranked 
G. Chauvin et al.: The VLT/NaCo large program to probe the occurrence of exoplanets in wide orbits. II.

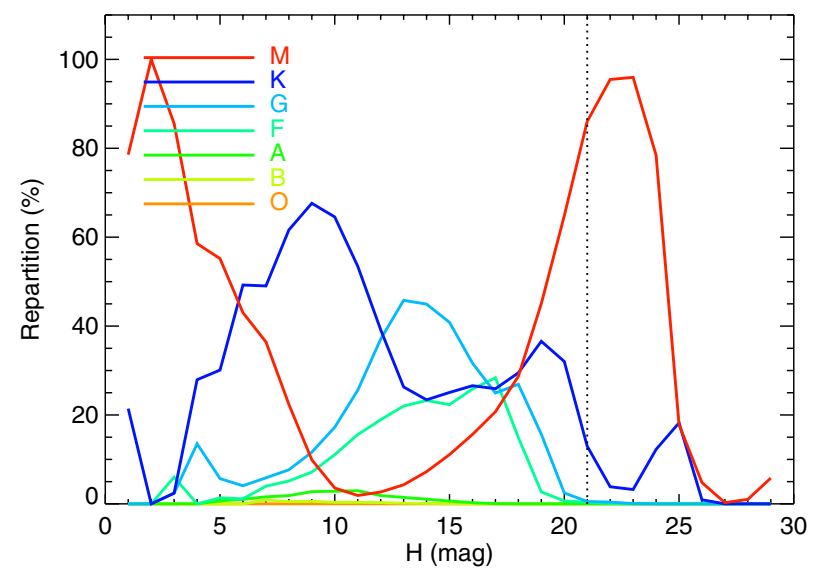

Fig. 5. Expected spectral type distribution of field stars from the Besançon galactic population model, as observed during the NaCo-LP. The FoV, the typical magnitude limit of the NaCo-LP $\left(H_{\mathrm{lim}}=21 \mathrm{mag}\right)$, and the galactic coordinates of all targets were considered. The predicted repartion is given as a function of the spectral type and the apparent magnitude in $H$-band.

by priority as a function of their predicted masses (higher priority to lower masses), projected physical separations (assuming they would be bound; higher priority to closer candidates) and predicted false alarm probabilities using the Besançon galactic population model (Robin et al. 2003) to guide the follow-up strategy. Follow-up observations with a second epoch were obtained for 29 targets, including the Moth system (HD 61005) that characterized during dedicated follow-up observations. The amplitude of stellar proper motion (larger than $30 \mathrm{mas} / \mathrm{yr}$ for $80 \%$ of the NaCo-LP target) enabled a rapid identification over a $1 \mathrm{yr}$ interval (see Fig. 1, bottom-middle).

For the 29 systems with at least 2-epoch observations (including the Moth system), we used a $\chi^{2}$ probability test with $2 \times N_{\text {epochs }}$ degrees of freedom (corresponding to the measurements: separations in the $\Delta \alpha$ and $\Delta \delta$ directions for the number $N_{\text {epochs }}$ of epochs). This test considers the uncertainties in the relative positions measured at each epoch and the uncertainties in the primary proper motion and parallax (or distance). Figure 6 gives an illustration of a $(\Delta \alpha, \Delta \delta)$ diagram that was used to identify a stationary background contaminant around TYC 7617-0549-1. A status has been assigned to each candidate as a background contaminant (B; $P_{\text {comoving, } \chi^{2}}<1 \%$ and with a relative motion compatible with a background source), comoving $\left(\mathrm{C} ; P_{\mathrm{BKG}, \chi^{2}}<1 \%\right)$ and with the relative motion compatible with a comoving companion), and undefined (U) when observed at only one epoch or when not satisfying the first two classifications.

Only one comoving companion, the white dwarf companion around HD 8049 described hereafter, was identified. Among the 28 other follow-up fields, ten fields have been completely characterized, and 18 are partially due to detection limits variation from one epoch to another. Fourteen fields still require second epoch observations. The status of all the candidates is given in Table 6.

\subsection{A white dwarf companion around HD 8049}

The only comoving companion identified in this survey with a preliminary predicted mass of $35 M_{\text {Jup }}$ was discovered around the star HD $8049(\mathrm{~K} 2,33.6 \mathrm{pc})$. The star had a predicted age of 90-400 Myr from its rotational period, H\&K emission and $\mathrm{X}$-ray emission. Thanks to the high proper motion of the central

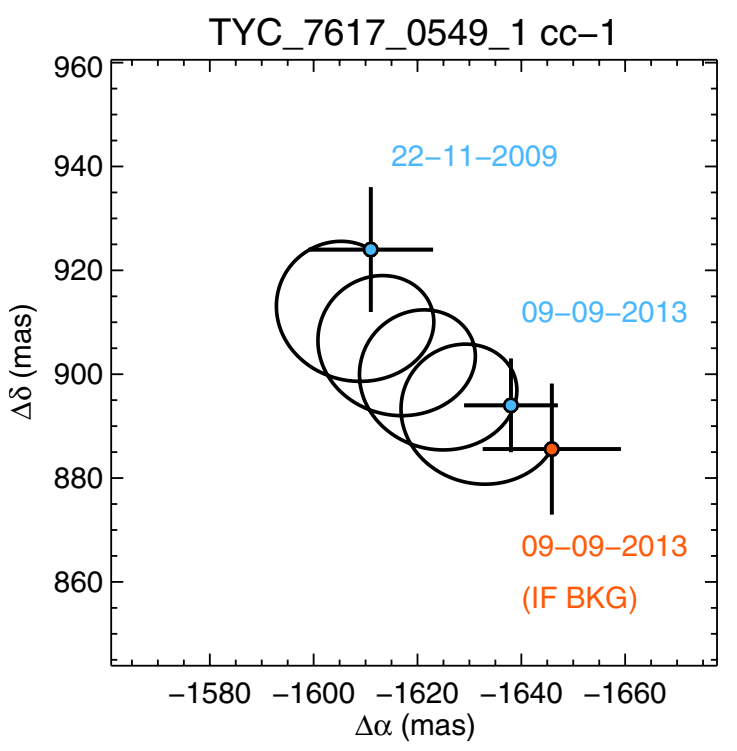

Fig. 6. VLT/NaCo measurements (filled circles with uncertainties) of the offset positions of the companion candidate to TYC 7617-0549-1 (see Fig. 3). The expected variation of offset positions, if the candidate is a background object, is shown (curved line). The variation is estimated based on the parallactic and proper motions of the primary star, as well as the initial offset position of the companion candidate from TYC 7617-0549-1. The companion candidate is clearly identified here as a stationary background contaminant.

$\operatorname{star}\left(\mu_{\alpha}=65.99 \pm 1.18 \mathrm{mas} / \mathrm{yr}\right.$ and $\left.\mu_{\delta}=240.99 \pm 0.98 \mathrm{mas} / \mathrm{yr}\right)$, a $\chi^{2}$ probability test on $\Delta \alpha$ and $\Delta \delta$ with respect to the star at two epochs rejected the possibility (at $99 \%$ certainty) that the object was a background source. Further analysis using archived data, radial velocity observations spanning a time range of $\sim 30 \mathrm{yr}, U$-band imaging with EFOSC, and near-infrared spectroscopy of the comoving companion with VLT/SINFONI finally revealed that the companion was actually a white dwarf (WD) with temperature $T_{\mathrm{eff}}=18800 \pm 2100 \mathrm{~K}$ and mass $M_{\mathrm{WD}}=0.56 \pm 0.08 M_{\odot}$.

This astrophysical false positive revealed that the system age was much older than initially thought. The age diagnostics have likely been affected, as the central star has been probably rejuvenated by the accretion of some amount of mass and angular momentum at the time of mass loss from the WD progenitor. A complete analysis of the system (evolution and kinematics) by Zurlo et al. (2013) actually reveals that the resulting age of the system to be about 3-6 Gyr.

\subsection{The Moth resolved as a thin debris-disk}

In the course of the survey, the emblematic star HD 61005 (G8V, $90 \mathrm{Myr}, 34.5 \mathrm{pc}$ ), known to host The Moth debris disk (Hines et al. 2007), was observed. The $\mathrm{NaCo} H$-band image remarkably resolves the disk component as a distinct narrow ring at inclination of $i=84.3 \pm 1.0^{\circ}$, with a semimajor-axis of $a=$ $61.25 \pm 0.85 \mathrm{AU}$ and an eccentricity of $e=0.045 \pm 0.015$. The observations also revealed that the the ring centre is offset from the star by at least $2.75 \pm 0.85 \mathrm{AU}$, which indicates a possibly dynamical perturbation by a planetary companion that perturbs the remnant planetesimal belt. The observations and the detailed disk modeling were published by Buenzli et al. (2010). Subsequent observations did not reveal any giant planet companions. Three other stars of our sample are known to host debris-disks: HIP 11360 (HD 15115; Kalas et al. 2007, Rodigas et al. 2012), HIP 99273 (HD 191089; Churcher et al. 2011), and 

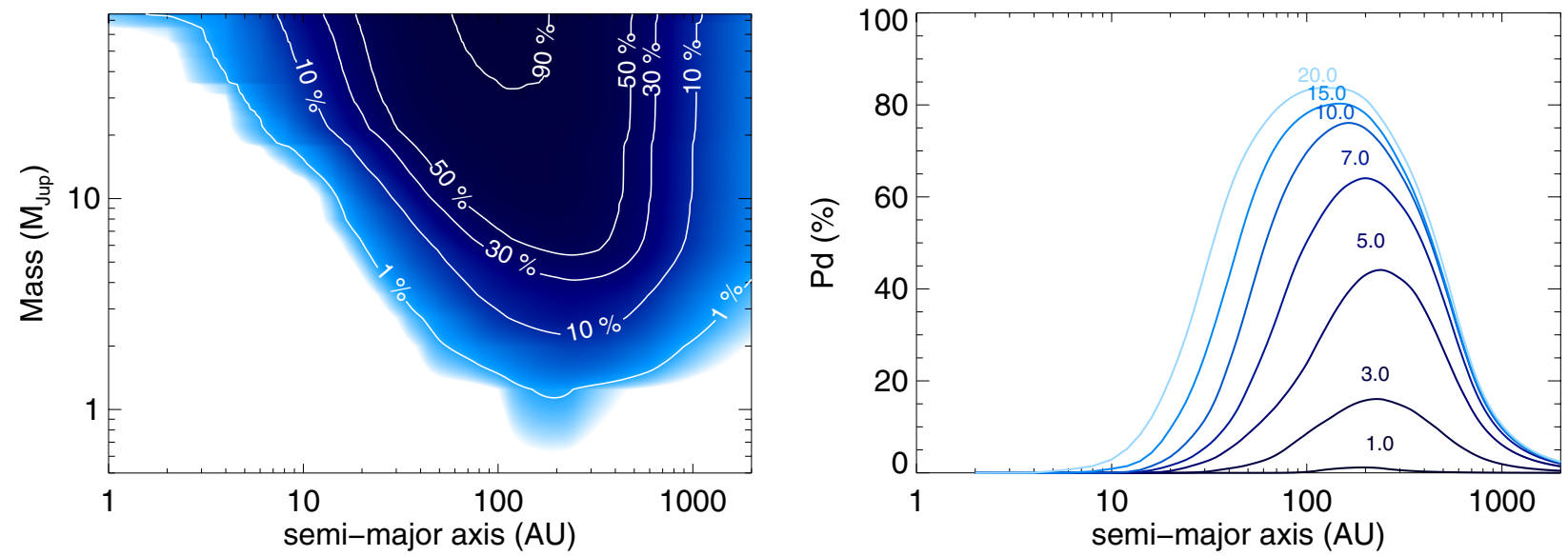

Fig. 7. Left: NaCo-LP mean detection probability map $\left(\left\langle p_{j}\right\rangle\right)$ as a function of the mass and semi-major axis. Right: mean probability curves for different masses $\left(1,3,5,7,10,15\right.$, and $\left.20 M_{\mathrm{Jup}}\right)$ as a function of the semi-major axis.

HIP 76829 (HD 139664; Kalas et al. 2006). No clear detection was obtained with our ADI analysis.

\section{Statistical analysis}

\subsection{Sample definition}

To define a meaningful sample for the statistical analysis of the survey, we first removed all visual and spectroscopic binaries from the sample of 76 stars observed in ADI. It includes the six visual multiple systems observed in that mode (TYC 0603-0461-1, TYC 7835-2569-1, HD 8049, HIP 8290, TYC 8927-3620-1 and TYC 8989-0583-1), and seven new spectroscopic binaries unknown at the time of our sample selection. We have then selected two sub-samples:

- the full-stat sample of 63 stars that includes all single stars observed in ADI with detection sensitivities down to planetary masses for physical separations ranging from 10 to $2000 \mathrm{AU}$. The status of all the candidates detected in these fields have, however, not been fully completed, although a large majority are expected to be stationary background contaminants. This sample gives an estimation of the ultimate performances of the survey in terms of masses and physical separations, when the candidate status identification will be complete, which is probably with SPHERE in the forthcoming years;

- the complete-stat sample of 51 stars has been restrained to all systems for which the candidate status identification up to $300 \mathrm{AU}$ was complete. This includes cases with no companion candidates detected or with companion candidates properly identified thanks to our follow-up observations as stationary background sources or comoving companions. In the case of follow-up observations with variable detection performances from one epoch to another (therefore with possible undefined faint sources due to the lack of redetection), only the worst detection limit was considered. These selection criteria offered us a meaningful sample at the end for which the detection and the status identification of the candidates was complete.

\subsection{Survey detection probability}

To correct for the projection effect from the observations, we then ran a set of Monte-Carlo simulations using an optimized version of the MESS code (Bonavita et al. 2012). For the full-stat sample, the code generates a uniform grid of mass (with a sampling of $0.5 M_{\text {Jup }}$ in the $[1,75] M_{\text {Jup }}$ interval), and semi-major axis (with a sampling of 1 AU between 1 and $1000 \mathrm{AU}$, and $2 \mathrm{AU}$ between 1000 and $2000 \mathrm{AU}$ for the [1, 2000] AU interval). For the complete-stat sample, the uniform grid is generated in the semi-major axis ranges between $[1,300]$ AU with a sampling of $1 \mathrm{AU}$. For each point in the grid, 100 orbits were generated and randomly oriented in space from uniform distributions in $\sin (i), \omega, \Omega, e \leq 0.8$, and $T_{\mathrm{p}}$. The on-sky projected position (separation and position angle) at the time of the observation is then computed for each orbit and compared to our $5 \sigma$ 2D-detection maps to determine the individual detection probability $\left(p_{j}\right)$ of planets around each star. The average of all individual detection limits gives us the typical mean detection probability $\left(\left\langle p_{j}\right\rangle\right)$ of the NaCo-LP to the planet and BD companion population. The results for the full-stat and complete-stat samples are shown in Figs. 7 and 8 top) respectively. The detection probabilities in both cases do not significantly differ at less than 300 AU. Most companions more massive than $20 M_{\text {Jup }}$ with a semi-major axis between 70 and $200 \mathrm{AU}$ should have been detected during our survey. We are $50 \%$ sensitive to massive ( $\geq 10 M_{\text {Jup }}$ ) planets and brown dwarfs with a semi-major axis between 60 and 400 AU. Finally, the detection of giant planets as light as $5 M_{\text {Jup }}$ between $50-800 \mathrm{AU}$ is only possible for $10 \%$ of the stars observed. The relatively small number of very young stars (see Fig. 1) is responsible for this limited sensitivity to light giant planets.

\subsection{Giant planet occurrence at wide orbits}

To derive the occurrence of giant planets and brown dwarfs in our survey, we only considered the complete-stat sample with a complete census of the candidates status within 300 AU. As no planetary mass or brown dwarf companions were detected, we considered here a null-detection result. We then used the mean detection probability $\left(\left\langle p_{j}\right\rangle\right)$ to derive the giant planet and brown dwarf occurrence upper limit $\left(f_{\max }\right)$ that is compatible with the survey detection limits. The probability of planet detection for a survey of $N$ stars is described by a binomial distribution, given a success probability $f p_{j}$ with $f$ as the fraction of stars with planets. The parameter $p_{j}$ is the individual detection probability of detecting a planet if it is present around the star $j$ and computed previously. Assuming that the number of expected detected planets is small compared to the number of stars observed, the binomial distribution can be approximated by a Poisson distribution 
G. Chauvin et al.: The VLT/NaCo large program to probe the occurrence of exoplanets in wide orbits. II.
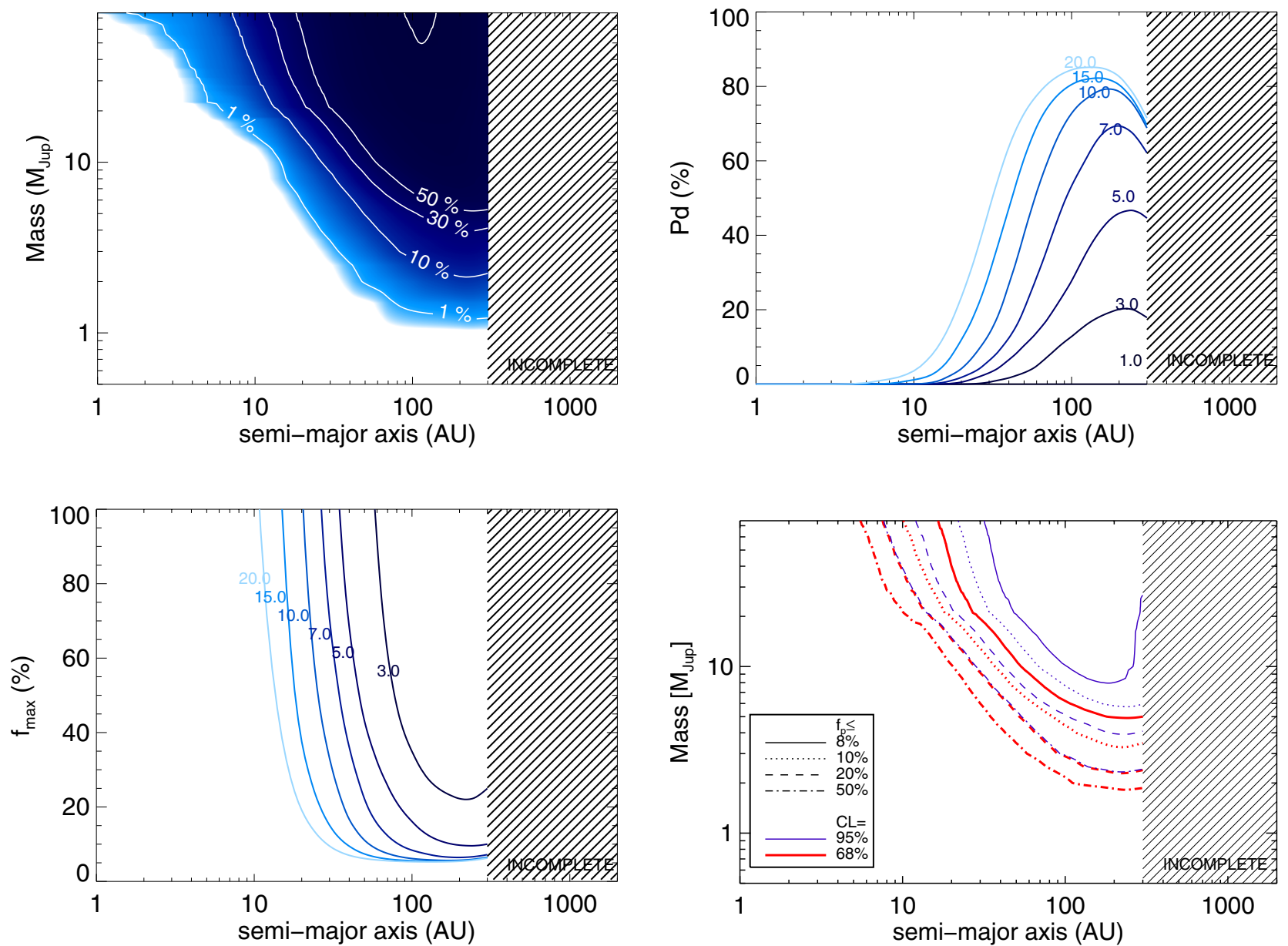

Fig. 8. Results for the complete-stat sample. Top left: NaCo-LP mean detection probability map $\left(\left\langle p_{j}\right\rangle\right)$ as a function of the mass and semi-major axis. Top right: mean probability curves for different masses $\left(1,3,5,7,10,15\right.$, and $\left.20 M_{\text {Jup }}\right)$ as a function of the semi-major axis. Bottom left: giant planet and brown dwarf occurrence upper limit $\left(f_{\max }\right)$, considering a 95\% confidence level, for different masses $\left(3,5,7,10,15\right.$, and $\left.20 M_{\mathrm{Jup}}\right)$ as a function of the semi-major axis considering the null-detection result and an uniform distribution of planets and brown dwarfs in terms of masses and semi-major axis. Bottom right: same occurrence upper limit $\left(f_{\max }\right)$ expressed this time in a mass versus semi-major axis diagramme for a $68 \%$ and 95\% confidence level (following Biller et al. 2007; Nielsen et al. 2008 representation).

to derive a simple analytical solution for the exoplanet fraction upper limit $\left(f_{\max }\right)$. The formalism is described by Carson et al. (2006) and Lafrenière et al. (2007). The result is shown in Fig. 8 (bottom-left and bottom-bight). For this complete-stat sample, we constrain the occurrence of exoplanets that are more massive than $5 M_{\text {Jup }}$ to typically less than $15 \%$ between 100 and $300 \mathrm{AU}$. The occurence is less than $10 \%$ between 50 and 300 AU for exoplanets that are more massive than $10 \mathrm{M}_{\mathrm{Jup}}$. We consider here a uniform input distribution with a confidence level of $95 \%$. These values are consistent with current estimations from various studies with comparable sensitivities around young, solar-type stars $\left(f_{\max } \leq 9.7 \%\right.$ for $[0.5,13] M_{\text {Jup }}$ planet between [50-250] AU by Lafrenière et al. 2007; $f_{\max } \leq 10 \%$ for $[1,13] M_{\text {Jup }}$ planet between [40-150] AU by Chauvin et al. 2010; $f_{\max } \leq 6 \%$ for $[1,20] M_{\text {Jup }}$ planet between [10-150] AU by Biller et al. 2013).

A more complete analysis, which combines the results of the NaCo-LP with archive data for a total of $\sim 210$ observed stars in direct imaging, will be presented in related papers by Vigan et al. (in prep.) and Reggiani et al. (in prep.). This analysis will provide significant and relevant statistical constraints on the population of planets and brown dwarfs around young, nearby solartype (FGK) stars (single or members of wide binaries) and enable tests of planet and brown dwarf formation models.

\section{Conclusion}

In the context of the scientific preparation of the VLT/SPHERE guaranteed time, we have conducted a survey of 86 young, close and mostly solar-type stars by using NaCo at the VLT between 2009 and 2013. Our main goals were to detect new giant planets and brown dwarf companions and to initiate a relevant statistical study of their occurrence at wide (10-2000 AU) orbits. The NaCo instrument was used in pupil-stabilized mode to perform angular differential imaging at $H$-band. It enables us to reach contrast performances as small as $10^{-6}$ at $1.5^{\prime \prime}$. Of the 86 stars observed, the survey led to

- the discovery of 11 new close binaries that we characterized in terms of relative photometry and astrometry;

- the detection of more than 700 companion candidates with $90 \%$ of them being located in six crowded fields. Among the 76 stars observed in deep ADI, 33 systems have no point-source detected in their vicinity, and 43 systems have at least one companion candidate detected. Repeated observations at several epochs enabled us to analyze the candidate status, either completely or partially, around 29 stars. Planetary mass candidates with proper follow-up were all 
identified as background sources. Additional follow-up observations are still necessary to fully complete the status identification of all candidates detected in the survey owing to the variability of the detection performances from one run to another. It shows that more than two epochs are generally necessary during a survey for a full exploration of the companions content.

- The discovery of a unique comoving companion to the star HD 8049. This result has been published by Zurlo et al. (2013) and has revealed that the companion was actually a white dwarf with temperature $T_{\text {eff }}=18800 \pm 2100 \mathrm{~K}$ and mass $M_{\mathrm{WD}}=0.56 \pm 0.08 M_{\odot}$.

- New high-contrast images of the Moth debris-disk at HD 61005. The NaCo $H$-band image remarkably resolves the disk component as a distinct narrow ring offset from the star by at least $2.75 \pm 0.85 \mathrm{AU}$, which indicates a possibly dynamical perturbation by a planetary companion. This study was published by Buenzli et al. (2010).

- Finally, a preliminary statistical analysis of the survey detection probabitlities around the sample of 63 young, single and mostly solar-type (FGK) stars observed in angular differential imaging with detection performances enabling the search for planets and brown dwarfs in the stellar environment. Most companions that are more massive than $20 M_{\text {Jup }}$ with a semi-major axis between 70 and $200 \mathrm{AU}$ should have been detected during our survey. We are $50 \%$ sensitive to massive $\left(\geq 10 M_{\text {Jup }}\right)$ planets and brown dwarfs with a semimajor axis between 60 and 400 AU. Finally, the detection of giant planets as light as $5 M_{\text {Jup }}$ between 50-800 AU is only possible for $10 \%$ of the stars observed. We have then defined a more complete sample of 51 stars restrained to all systems for which the candidate status identification was complete up to $300 \mathrm{AU}$. This includes cases with no companion candidates detected or with companion candidates properly and completely identified. Based on this complete sample average detection probability, a non-detection result, and the consideration of a uniform distribution of giant planets and brown dwarf companions in terms of semi-major axis and mass, we derive a typical upper limit for the occurrence of exoplanets that are more massive than $5 M_{\text {Jup }}$ of $15 \%$ between 100 and $300 \mathrm{AU}$, and a limit of $10 \%$ between 50 and $300 \mathrm{AU}$ for EPs that are more massive than $10 M_{\text {Jup }}$ with a confidence level of $95 \%$.

Combined with compiled archived data, the results of this survey offer a unique sample of 210 young, solar-type stars that are observed in deep imaging as a mean to constrain the presence of giant planets and brown dwarfs in their close environment. A more complete statistical analysis will be published in two linked articles by Vigan et al. (in prep.) and Reggiani et al. (in prep.), which will test the relevance of various analytical distributions for describing the giant planet and brown dwarf companion population at wide orbits but will also bring further constraints on current theories of planetary formation. All final products of this survey (images, detection limits, and candidate status) will be released in the Deep Imaging Virtual Archive (DIVA) database with the archive data used for full statistical analysis. We encourage the community to support this effort by sharing the final products (reduced images, detection limits, and candidate relative astrometry, photometry, and status) of their published surveys to optimally prepare the future of planet imaging searches that come with the new generation of planet imagers like LMIRCam, MagAO, SPHERE, GPI, and SCExAO. In the long term, these include JWST (Clampin 2010), TMT-PFI
(Simard et al. 2010), and the EELT instruments (METIS or EMIDIR, Brandl et al. 2010; EPICS or E-PCS, Kasper et al. 2010).

Acknowledgements. We greatly thank the staff of ESO-VLT for their support at the telescope. This publication has made use of the SIMBAD and VizieR database operated at CDS, Strasbourg, France. Finally, we acknowledge supports from: 1) the French National Research Agency (ANR) through project grant ANR10-BLANC0504-01, the CNRS-D2P PICS grant, and the Programmes Nationaux de Planétologie et de Physique Stellaire (PNP \& PNPS), in France for G.C., A.V, P.D., J.-L.B., A.-M.L. and D.M.; 2) INAF through the PRININAF 2010 Planetary Systems at Young Ages project grant for S.D., D.M., M.B. and R.G. and 3) the US National Science Foundation under Award No. 1009203 for J.C.

\section{References}

Alibert, Y., Mordasini, C., \& Benz, W. 2004, A\&A, 417, L25

Apai, D., Janson, M., Moro-Martín, A., et al. 2008, ApJ, 672, 1196

Baraffe, I., Chabrier, G., \& Gallardo, J. 2009, A\&A, 702, 27

Barman, T. S., Macintosh, B., Konopacky, Q. M., \& Marois, C. 2011a, ApJ, 733,

Barman, T. S., Macintosh, B., Konopacky, Q. M., \& Marois, C. 2011b, ApJ, 735 , L 39

Batalha, N. M., Rowe, J. F., Bryson, S. T., et al. 2013, ApJS, 204, 24

Beuzit, J.-L., Feldt, M., Dohlen, K., et al. 2008, SPIE, 7014, 41

Biller, B. A., Close, L. M., Masciadri, E., et al. 2007, ApJS, 173, 143

Biller, E., Liu, M., Wahhaj, Z., et al. 2013, ApJ, 777, 160

Boley, A. C. 2009, ApJ, 695, L53

Boley, A. C., Hayfield, T., Mayer, L., \& Durisen, R. H. 2010, Icarus, 207, 509

Bonavita, M., Chauvin, G., Desidera, S., et al. 2012, A\&A, 537, A67

Bonfils, X., Delfosse, X., Udry, S., et al. 2013, A\&A, 549, A109

Bonnefoy, M., Chauvin, G., Lagrange, A. M., et al. 2010, A\&A, 512, A52

Bonnefoy, M., Boccaletti, A., Lagrange, A. M., et al. 2013, A\&A, 555, A107

Bonnefoy, M., Chauvin G., Lagrange, A. M., et al. 2014a, A\&A, 562, A127

Bonnefoy, M., Currie, M., Marleau, G. D., et al. 2014b, A\&A, 562, A111

Boss, A. P. 1997, Science, 276, 1836

Bowler, B., Liu, M., Dupuy, T. J., \& Cushing, M. C. 2010, ApJ, 723, 850

Brandl, B. R., Lenzen, R., Pantin, E., et al. 2010, SPIE, 7735, 83

Brandner, W., Alcala, J. M., Kunkel, M., Moneti, A., \& Zinnecker, H. 1996

A\&A, 307, 121

Brandt, T., Kuzuhara, M., McElwain, M. W., et al. 2013, ApJ, 786, 1

Buenzli, E., Thalmann, C., Vigan, A., et al. 2010, A\&A, 524, L1

Carson, J., Eikenberry, S. S., Smith, J. J., \& Cordes, J. M. 2006, AJ, 132, 1146

Carson, J., Thalmann, C., Janson, M., et al. 2013, ApJ, 763, L32

Chauvin, G., Thomson, M., Dumas, C., et al. 2003, A\&A, 404, 157

Chauvin, G., Lagrange, A.-M., Zuckerman, B., et al. 2005a, A\&A, 438, L29

Chauvin, G., Lagrange, A.-M., Dumas, C., et al. 2005b, A\&A, 438, L25

Chauvin, G., Lagrange, A.-M., Bonavita, M., et al. 2010, A\&A, 509, A52

Chauvin, G., Lagrange, A.-M., Beust, H., et al., 2012, A\&A, 542, A41

Churcher, L., Wyatt, M., \& Smith, R. 2011, MNRAS, 410, 2

Clampin, M. 2010, in Pathways Towards Habitable Planets, ASP Conf. Ser., 430, 167

Close, L., Males, J. R., Kopon, D. A., et al. 2012, SPIE, 8447, 0

Delorme, P., Lagrange, A.-M., Chauvin, G., et al. 2012, A\&A, 539, A72

Desidera S., Covino E., Messina S., et al. 2015, A\&A, 573, A126

Devillar, N. 1997, The Messenger, 87, 19

Esposito, S., Mesa, D., Skemer, A., et al. 2013, A\&A, 549, A52

Dawson, R. I., \& Murray-Clay, R. A. 2013, ApJ, 767, L24

Forgan, D., \& Rice, K. 2013, MNRAS, 432, 3168

Guyon, O., Martinache, F., Garrel, V., et al. 2010, SPIE, 7736, 71

Heinze, A. N., Hinz, P. M., Sivanandam, S., et al. 2010a, ApJ, 714, 1551

Heinze, A. N., Hinz, P. M., Kenworthy, M., et al. 2010b, ApJ, 714, 1570

Hines, D. C., Schneider, G., Hollenbach, D., et al. 2007, ApJ, 671, L165

Hoff, W., Henning, T., \& Pfau, W. 1998, A\&A, 336, 242

Howard, A. W., Johnson, J. A., \& Marcy, G. W. 2010, ApJ, 721, 1467

Howard, A. W., Marcy, G. W., \& Bryson, S. T. 2012, ApJ, 201, 15

Janson, M., Bergfors, C., Goto, M., Brandner, W., \& Lafrenière, D. 2010, ApJ 710, L35

Janson, M., Bonavita, M., Klahr, H., et al. 2013a, ApJ, 736, 89

Janson, M., Brandt, T. D., Moro-Martín, A., et al. 2013b, ApJ, 773, 73

Joergens, V., Bonnefoy, M., Liu, Y., et al. 2013, A\&A, 558, L7

Kalas, P., Graham, J. R., Clampin, M. C., \& Fitzgerald, M. P. 2006, ApJ, 637, L57 
G. Chauvin et al.: The VLT/NaCo large program to probe the occurrence of exoplanets in wide orbits. II.

Kalas, P., Fitzgerald, M. P., \& Graham, J. R. 2007, ApJ, 661, L85

Kalas, P., Graham, J. R., Chiang, E., et al. 2008, Science, 322, 1345

Kalas, P., Graham, J. R., Fitzgerald, M. P., \& Clampin, M. 2013, ApJ, 775, 56

Kasper, M., Apai, D., Janson, M., \& Brandner, W. 2007, A\&A, 472, 321

Kasper, M., Beuzit, J.-L., Vérinaud, C., et al. 2010, SPIE, 7735, 81

Kastner, J. H., Zuckerman, B., Weintraub, D. A., \& Forveille, T. 1997, Science, 277,67

Kley, W., \& Nelson, R. P. 2012, ARA\&A, 50, 211

Köhler, R., Kunkel, M., Leinert, C., \& Zinnecker, H. 2000, A\&A, 356, 541

Konopacky, Q. M., Barman, T. S., Macintosh, B. A., \& Marois, C. 2013, Science, 339,1398

Kuzuhara, M., Tamura, M., Kudo, T., et al. 2013, ApJ, 774, 11

Lafrenière, D., Doyon, R., Marois, C., et al. 2007, ApJ, 670, 1367

Lafrenière, D., Jayawardhana, R., van Kerkwijk, M. H., et al. 2008, ApJ, 689, 153

Lagrange, A.-M., Gratadour, D., Chauvin, G., et al. 2009, A\&A, 506, L972

Lambrechts, M., \& Johansen, A. 2012, A\&A, 544, L32

Lenzen, R., Hartung, M., Brandner, et al. 2002, SPIE, 4841

Liu, M. C., Magnier, E. A., Deacon, N. R., et al. 2013, ApJ, 777, L20

Lowrance, P. J., Becklin, E. E., Schneider, G., et al. 2005, AJ, 130, 1845

Luhman, K. 2012, ARA\&A, 50, 65

Macintosh, B., Graham, J. R., Palmer, D., et al. 2008, SPIE, 7015, 31

Marois, C., Lafrenière, D., Doyon, R., Macintosh, B., \& Nadeau, D. 2006, ApJ, 641, 556

Marois, C., Macintosh, B., Barman, T., et al. 2008, Science, 322, 1348

Marois, C., Zuckerman, B., Konopacky, Q. M., Macinosh, B., \& Barman, T. 2010, Nature 468, 1080

Masciadri, E., Mundt, R., Henning, Th., \& Alvarez, C. 2005, ApJ, 625, 1004

Mayor, M., Marmier, M., Lovis, C., et al. 2011, A\&A, submitted [arXiv: 1109.2497]

McCaughrean, M. J., \& Stauffer, J. R. 1994, AJ, 108, 1382
Morbidelli, A., \& Nesvorny, D. 2012, A\&A, 546, A18

Naoz, S., Farr, W. M., Lithwick, Y., Rasio, F. A., \& Teyssandier, J. 2011, Nature, 473,187

Nayakshin, S. 2010, MNRAS, 402, 789

Neuhäuser, R., Guenther, E. W., Alves, J., et al. 2003, Astron. Nachr., 324, 535

Nielsen, E., Liu, M., Wahhaj, Z., et al. 2013, ApJ, 776, 4

Pollack, J. B., Hubickyj, O., Bodenheimer, P., et al. 1996, Icarus, 124, 62

Rameau, J., Chauvin, G., Lagrange, A.-M., et al. 2013a, ApJ, 772, L15

Rameau, J., Chauvin, G., Lagrange, A.-M., et al. 2013b, ApJ, 779, L26

Rameau, J., Chauvin, G., Lagrange, A.-M., et al. 2013c, A\&A, 553, A60

Robin, A. C., Reylé, C., Derrière, S., \& Picaud, S. 2003, A\&A, 409, 523

Rodigas, T. J., Hinz, P. M., Leisenring, J., et al. 2012, ApJ, 752, 57

Rousset, G., Lacombe, F., Puget, P., et al. 2002, SPIE, 4007

Skrutskie, M. F., Jones, T., Hinz, P., et al. 2010, SPIE, 7735, 118

Simard, L., Crampton, D., Ellerbroek, B., \& Boyer, C. 2010, SPIE, 7735, 70

Stamatellos, D., \& Withworth, A. P. 2008, A\&A, 480, 879

Torres, C. A. O., Quast, G. R., Melo, C. H. F., \& Sterzik, M. F. 2008, in Handbook of Star Forming Regions, Vol. II: The Southern Sky ASP Monograph Publications, 5, 757

Udry, S., \& Santos, N. C. 2007, ARA\&A, 45, 397

van Dessel, E., \& Sinachopoulos, D. 1993, A\&AS, 100, 517

Vigan, A., Patience, J., Marois, C., et al. 2012, A\&A, 544, A9

Vorobyov, E. I. 2013, A\&A, 552, A129

Wahhaj, Z., Liu, M., Nielsen, E. L., et al. 2013, ApJ, 773, 179

Whitworth, A., Bate, M. R., Nordlund, A., Reipurth, B., \& Zinnecker, H. 2007, PPV Conf. (Tucson: University of Arizona Press), 951, 459

Wright, H. S., Marcy, G. W., Howard, A. W., et al. 2012, ApJ, 753, 160

Yamamoto, K., Matsuo, T., Shibai, H., et al. 2013, PASJ, 65, 90

Zapatero Osorio, M. R., Béjar, V. J. S., \& Martín, E. L. 2000, Science, 290, 103

Zuckerman, B., \& Song, I. 2004, ARA\&A, 42, 685

Zurlo, A., Vigan, A., Hagelberg, J., et al. 2013, A\&A, 554, A21 
Table 2. NaCo-LP target sample and properties.

\begin{tabular}{|c|c|c|c|c|c|c|c|c|}
\hline Name-1 & Name-2 & $\begin{array}{l}H \\
(\mathrm{mag})\end{array}$ & SpT & $\begin{array}{l}d \\
(\mathrm{pc})\end{array}$ & $\begin{array}{l}\text { Age } \\
\text { (Myr) }\end{array}$ & Binarity & Mode & Comments \\
\hline TYC 5839-0596-1 & BD-16-20 & 6.6 & K0IVe & 43.5 & 150. & SB2 & sat, ADI, H & \\
\hline TYC 0603-0461-1 & $\mathrm{BD}+07-85$ & 7.4 & $\mathrm{~K} 4 \mathrm{Ve}$ & 58.2 & 100. & Bin (new) & sat, ADI, H & \\
\hline HIP 3924 & HD 4944 & 6.7 & F7V & 53.2 & 500. & SB2 & sat, ADI, H & \\
\hline HIP 6177 & HD 8049 & 6.7 & $\mathrm{~K} 2 \mathrm{~V}$ & 33.6 & 3000. & & sat, ADI, H & $\mathrm{cc}$ \\
\hline HIP 8038 & HD 10611B & 7.2 & $\mathrm{~K} 5 \mathrm{Ve}$ & 29.3 & 150. & Bin (new) & nonsat, ADI, $\mathrm{H}$ & \\
\hline HIP 10602 & HD 14228 & 4.0 & B0V & 47.1 & 30. & & sat, ADI, H & \\
\hline HIP 11360 & HD 15115 & 5.9 & $\mathrm{~F} 2$ & 45.2 & 30. & & sat, ADI, H & \\
\hline TYC 8484-1507-1 & CD-53-535 & 6.6 & G8V & 60.5 & 100. & Bin (known) & nonsat, ADI, $\mathrm{H}$ & \\
\hline HIP 12394 & HD 16978 & 4.4 & B9III & 46.6 & 30. & & sat, ADI, H & \\
\hline HIP 13008 & HD 17438 & 5.5 & F2V & 39.6 & 1000. & & sat, ADI, H & $\mathrm{cc}$ \\
\hline HIP 14684 & IS-Eri & 6.8 & G0 & 37.4 & 100. & & sat, ADI, H & $\mathrm{cc}$ \\
\hline TYC 8060-1673-1 & CD-46-1064 & 7.2 & $\mathrm{~K} 3 \mathrm{~V}$ & 40.4 & 30. & & sat, ADI, H & \\
\hline HIP 19775 & HD 26980 & 7.7 & G3V & 80.5 & 30. & & sat, ADI, H & \\
\hline HIP 23316 & HD 32372 & 7.9 & G5V & 76.3 & 30. & & sat, ADI, H & \\
\hline HD 32981 & BD-16-1042 & 7.8 & F8V & 86.7 & 100. & & sat, ADI, H & \\
\hline BD-09-1108 & $\mathrm{xxxx}$ & 8.2 & G5 & 93.6 & 30. & & sat, ADI, H & \\
\hline HIP 25434 & HD 274197 & 7.9 & G0 & 79.1 & 20. & & sat, ADI, H & $\operatorname{ccs}$ \\
\hline TYC9162-0698-1 & HD 269620 & 8.2 & G6V & 77.7 & 30. & & sat, ADI, H & $\operatorname{ccs}$ \\
\hline TYC 5346-132-1 & BD-08-1195 & 8.1 & G7 & 81.2 & 30. & & sat, ADI, H & $\operatorname{ccs}$ \\
\hline HIP 30261 & HD 44748 & 7.6 & G6V & 61.8 & 100. & & sat, ADI, H & \\
\hline TYC 7617-0549-1 & CD-40-2458 & 8.2 & KOV & 77.8 & 30. & & sat, ADI, H & $\mathrm{cc}$ \\
\hline TYC 9181-0466-1 & HD 47875 & 7.4 & G4V & 77.7 & 30. & Bin (new) & nonsat, ADI, $\mathrm{H}$ & \\
\hline HIP 32235 & HD 49855 & 7.4 & G6V & 58.2 & 30. & & sat, ADI, H & $\mathrm{cc}$ \\
\hline HIP 35564 & HD 57852 & 5.1 & F2 & 31.7 & 200. & RV var & sat, ADI, H & $\operatorname{ccs}$ \\
\hline TYC 8128-1946-1 & CD-48-2972 & 8.1 & G8V & 89.7 & 45. & & sat, ADI, H & $\operatorname{ccs}$ \\
\hline HIP 36414 & HD 59704 & 6.5 & F7V & 52.5 & 200. & $\mathrm{SB}, \mathrm{RV}$ var & sat, ADI, H & $\operatorname{ccs}$ \\
\hline HIP 36948 & HD 61005 & 6.6 & G5V & 35.3 & 45. & & sat, ADI, H & $\operatorname{ccs}$ \\
\hline HIP 37563 & HD 62850 & 5.9 & G3V & 32.8 & 200. & & sat, ADI, H & \\
\hline HIP 37923 & HD 63608 & 6.5 & K0V & 36.8 & 200. & & sat, ADI, H & $\operatorname{ccs}$ \\
\hline TYC 8927-3620-1 & HD 77307 & 7.7 & G8IV & 81.8 & 20. & Bin (new) & sat, ADI, H & \\
\hline HIP 46634 & $\mathrm{BD}+11-2052 \mathrm{~B}$ & 6.8 & G5 & 42.3 & 300. & & sat, ADI, H & \\
\hline HIP 47646 & HD 84199 & 6.9 & F5V & 73.6 & 1150. & & sat, ADI, H & \\
\hline TWA-21 & HD 298936 & 7.3 & $\mathrm{~K} 3 \mathrm{Ve}$ & 54.8 & 17. & & sat, ADI, H & $\operatorname{ccs}$ \\
\hline TYC 7188-0575-1 & CD-31-8201 & 7.4 & KoVe & 43.2 & 150. & SB2 & sat, ADI, H & $\operatorname{ccs}$ \\
\hline TYC 6069-1214-1 & BD-19-3018 & 8.0 & KOV & 67.8 & 70. & & sat, ADI, H & \\
\hline TYC 7722-0207-1 & HD 296790 & 7.8 & $\mathrm{~K} 0 \mathrm{~V}$ & 65.8 & 100. & & sat, ADI, H & $\operatorname{ccs}$ \\
\hline TYC 7743-1091-1 & HD 99409 & 5.2 & G6III & 200.0 & 1700. & & sat, ADI, H & \\
\hline HIP 58240 & HD 103742 & 6.2 & G3V & 31.8 & 200. & & sat, ADI, H & $\mathrm{cc}$ \\
\hline TYC 9231-1566-1 & HD 105923 & 7.3 & G8V & 96.0 & 10. & Bin (new) & nonsat, ADI, $\mathrm{H}$ & \\
\hline TYC 8979-1683-1 & CD-62-657 & 7.5 & G7V & 75.6 & 17. & & sat, ADI, $\mathrm{H}$ & $\operatorname{ccs}$ \\
\hline TYC 8989-0583-1 & HD 112245 & 7.4 & KOVe & 65.4 & 17. & Bin (new) & sat, ADI, H & \\
\hline TYC 9245-0617-1 & CD-69-1055 & 7.7 & K0Ve & 93.0 & 10. & & sat, ADI, $\mathrm{H}$ & $\operatorname{ccs}$ \\
\hline HIP 63862 & HD 113553 & 6.8 & G5V & 49.0 & 150. & & sat, ADI, $\mathrm{H}$ & $\operatorname{ccs}$ \\
\hline TYC 7796-2110-1 & CD-41-7947 & 8.3 & $\mathrm{~K} 2 \mathrm{IVe}$ & 92.1 & 17. & & sat, ADI, H & $\operatorname{ccs}$ \\
\hline TYC 9010-1272-1 & HD 124831 & 7.8 & G3V & 86.5 & 30. & Bin (new) & nonsat, ADI, $\mathrm{H}$ & \\
\hline HIP 70351 & HD 125485 & 7.6 & G7V & 91.7 & 110. & & sat, ADI, $\mathrm{H}$ & $\operatorname{ccs}$ \\
\hline HIP 71908 & GJ-560A & 2.5 & F1V & 16.6 & 1110. & & sat, ADI, H & $\mathrm{cc}$ \\
\hline HIP 71933 & HD 129181 & 7.2 & F8V & 83.9 & 16. & & sat, ADI, H & $\operatorname{ccs}$ \\
\hline HIP 72399 & HD 130260A & 7.5 & $\mathrm{~K} 3 \mathrm{Ve}$ & 46.1 & 500. & SB1, RV var & sat, ADI, H & \\
\hline TYC 7835-2569-1 & HD 137059 & 7.1 & G3V & 70.2 & 120. & SB2 + Bin (known) & sat, ADI, H & \\
\hline HIP 76829 & HD 139664 & 3.7 & F5IV & 17.4 & 200. & & sat, ADI, H & $\operatorname{ccs}$ \\
\hline TYC 6781-0415-1 & CD-24-12231 & 7.4 & G9IVe & 106.0 & 11. & & sat, ADI, H & \\
\hline TYC 6786-0811-1 & CD-27-10549 & 7.5 & K0IV & 78.6 & 60. & Bin (known) & nonsat, ADI, $\mathrm{H}$ & \\
\hline HIP 78747 & HD 143928 & 5.1 & F3V & 37.9 & 1600. & & sat, ADI, H & $\operatorname{ccs}$ \\
\hline TYC 6209-0769-1 & BD-19-4341 & 7.4 & KOIV & 43.9 & 120. & & sat, ADI, H & $\mathrm{cc}$ \\
\hline HIP 79958 & HD 146464 & 6.7 & $\mathrm{~K} 3 \mathrm{Ve}$ & 27.2 & 130. & & sat, ADI, H & $\operatorname{ccs}$ \\
\hline HIP 80290 & HD 147491 & 8.0 & G2IV & 83.3 & 30. & Bin (new) & sat, ADI, H & $\operatorname{ccs}$ \\
\hline HIP 80758 & HD 148440 & 8.0 & G9Ve & 98.2 & 20. & & sat, ADI, H & $\operatorname{ccs}$ \\
\hline
\end{tabular}

Notes. In addition to the target name, $H$-band magnitude, spectral type, distance, and age, we have reported the multiplicity status with a flag (Bin for visual binaries with the indication that they are new or known, SB for spectroscopic binaries, RV var for radial velocity variable), the observing mode (nonsat for non-saturated or sat for saturated, ADI for angular differential imaging and the filter) and the presence of companion candidates (ccs). 
G. Chauvin et al.: The VLT/NaCo large program to probe the occurrence of exoplanets in wide orbits. II.

Table 2. continued.

\begin{tabular}{|c|c|c|c|c|c|c|c|c|}
\hline Name-1 & Name-2 & $\begin{array}{l}H \\
(\mathrm{mag})\end{array}$ & $\mathrm{SpT}$ & $\begin{array}{l}d \\
(\mathrm{pc})\end{array}$ & $\begin{array}{l}\text { Age } \\
\text { (Myr) }\end{array}$ & Binarity & Mode & Comments \\
\hline TYC 6818-1336-1 & HD 153439 & 7.8 & G0IV & 89.5 & 30. & & sat, ADI, H & $\operatorname{ccs}$ \\
\hline TYC 6815-0084-1 & CD-25-11942 & 7.7 & K0IV & 92.0 & 11. & & sat, ADI, H & $\operatorname{ccs}$ \\
\hline TYC 6815-0874-1 & CD-25-11922 & 10.1 & G2IV & 109.0 & 20 & SB2? & sat, ADI, H & $\operatorname{ccs}$ \\
\hline TYC 7362-0724-1 & HD 156097 & 7.8 & G5V & 90.0 & 20. & & sat, ADI, H & $\operatorname{ccs}$ \\
\hline TYC 8728-2262-1 & CD-54-7336 & 7.5 & $\mathrm{~K} 1 \mathrm{~V}$ & 70.4 & 12. & & sat, ADI, H & $\operatorname{ccs}$ \\
\hline HIP 86672 & HD 160682 & 7.4 & G5V & 78.0 & 30. & & sat, ADI, $\mathrm{H}$ & $\operatorname{ccs}$ \\
\hline HIP 89829 & HD 168210 & 7.2 & G5V & 72.6 & 16. & & sat, ADI, H & $\operatorname{ccs}$ \\
\hline HIP 93375 & HD 176367 & 7.3 & G1V & 58.8 & 100. & & sat, ADI, H & $\operatorname{ccs}$ \\
\hline HIP 94235 & HD 178085 & 7.0 & G1V & 61.3 & 100. & Bin (new) & nonsat, ADI, $\mathrm{H}$ & \\
\hline TYC 6893-1391-1 & CD-25-14224 & 7.8 & $\mathrm{~K} 2 \mathrm{~V}$ & 55.1 & 160. & & sat, ADI, $\mathrm{H}$ & $\operatorname{ccs}$ \\
\hline TYC 5206-0915-1 & BD-07-5533 & 8.2 & K1IV & 76.4 & 300. & & sat, ADI, H & \\
\hline TYC 5736-0649-1 & BD-14-5534 & 8.0 & G6V & 86.4 & 30. & & sat, ADI, H & $\operatorname{ccs}$ \\
\hline HD 189285 & BD-04-4987 & 8.0 & G5 & 77.8 & 100. & & sat, ADI, H & $\mathrm{cc}$ \\
\hline HIP 98470 & HD 189245 & 4.6 & F7V & 21.2 & 100. & & sat, ADI, H & \\
\hline TYC 5164-567-1 & BD-03-4778 & 8.0 & & 63.3 & 100. & & sat, ADI, H & $\operatorname{ccs}$ \\
\hline HIP 99273 & HD 191089 & 6.1 & F5V & 52.2 & 16. & & sat, ADI, H & \\
\hline HD 199058 & $\mathrm{BD}+08-4561$ & 7.0 & G5 & 66.2 & 100. & Bin (new) & nonsat, ADI, $\mathrm{H}$ & \\
\hline HIP 105384 & HD 203019 & 6.4 & $\mathrm{~K} 5 \mathrm{~V}$ & 35.0 & 400. & & sat, ADI, H & $\mathrm{cc}$ \\
\hline HIP 105612 & HD 202732 & 6.3 & G5V & 32.8 & 600. & & sat, ADI, H & \\
\hline HIP 107684 & HD 207278 & 8.1 & G7V & 90.2 & 100. & Bin (new) & nonsat, ADI, $\mathrm{H}$ & \\
\hline HIP 108422 & HD 208233 & 6.9 & G9IV & 58.0 & 30. & Bin (known) & nonsat, $\mathrm{ADI}, \mathrm{H}$ & \\
\hline TYC 8004-0083-1 & CD-40-14901 & 7.9 & G5V & 74.9 & 100. & & sat, ADI, H & \\
\hline HIP 114046 & HD 217897 & 3.6 & $\mathrm{M} 2 \mathrm{~V}$ & 3.3 & 8000. & & sat, ADI, H & \\
\hline TYC 9338-2016-1 & HD 220054 & 8.3 & G8V & 99.6 & 30. & & sat, ADI, H & \\
\hline TYC 9529-0340-1 & CD-86-147 & 7.6 & G8IV & 68.8 & 30. & & sat, ADI, H & \\
\hline TYC 9339-2158-1 & CD-69-2101 & 6.8 & $\mathrm{~K} 3 \mathrm{~V}$ & 30.6 & 300. & & sat, ADI, H & \\
\hline TYC 6406-0180-1 & HD 221545 & 7.7 & $\mathrm{~K} 0 \mathrm{~V}$ & 58.0 & 200. & & sat, ADI, H & \\
\hline HIP 116910 & HD 222575 & 7.8 & G8V & 63.7 & 100. & & sat, ADI, H & \\
\hline
\end{tabular}


A\&A 573, A127 (2015)

Table 6. Companion candidate characterization and identification (for multi-epoch observations).

\begin{tabular}{|c|c|c|c|c|c|c|c|}
\hline Name-1 & UT-Date & Candidate & $\begin{array}{l}\text { Sep } \\
\text { (mas) }\end{array}$ & $\begin{array}{l}\text { PA } \\
(\mathrm{deg})\end{array}$ & $\begin{array}{l}\Delta H \\
(\mathrm{mag})\end{array}$ & Status & Comments \\
\hline TYC 5839-0596-1 & 2009-11-24 & none & & & & & SB2 \\
\hline TYC 0603-0461-1 & 2009-11-24 & none & & & & & New binary (see Table 5) \\
\hline HIP 3924 & 2009-11-22 & none & & & & & SB2 \\
\hline \multirow[t]{2}{*}{ HIP 6177} & 2010-07-31 & cc-1 & $1566 \pm 6$ & $118.4 \pm 0.2$ & $7.1 \pm 0.1$ & & \\
\hline & 2011-07-28 & cc-1 & $1565 \pm 10$ & $118.0 \pm 0.4$ & & $\mathrm{C}$ & White dwarf companion \\
\hline HIP 8038 & $2010-07-31$ & none & & & & & New binary (see Table 5) \\
\hline \multirow[t]{2}{*}{ HIP 10602} & 2009-11-24 & none & & & & & A few exposures \\
\hline & 2010-07-30 & none & & & & & \\
\hline HIP 11360 & $2009-11-23$ & none & & & & & \\
\hline TYC 8484-1507-1 & 2010-07-31 & none & & & & & $\begin{array}{l}\text { Known }\left(\sim 8.6^{\prime \prime}\right) \text { binary } \\
\text { resolved by } 2 \text { MASS }\end{array}$ \\
\hline HIP 12394 & $2009-11-22$ & none & & & & & \\
\hline HIP 13008 & 2011-09-29 & cc-1 & $1710 \pm 7$ & $347.3 \pm 0.2$ & $6.9 \pm 0.0$ & $\mathrm{U}$ & \\
\hline \multirow[t]{2}{*}{ HIP 14684} & 2010-07-30 & cc-1 & $5454 \pm 13$ & $150.6 \pm 0.1$ & $10.7 \pm 0.1$ & $\mathrm{~B}$ & \\
\hline & $2011-12-23$ & cc-1 & $5274 \pm 6$ & $150.3 \pm 0.1$ & $11.1 \pm 0.1$ & B & \\
\hline TYC 8060-1673-1 & 2009-11-23 & none & & & & & \\
\hline HIP 19775 & 2009-11-22 & none & & & & & \\
\hline HIP 23316 & $2009-11-23$ & none & & & & & \\
\hline HD 32981 & 2009-11-24 & none & & & & & \\
\hline BD-09-1108 & 2009-11-22 & none & & & & & \\
\hline \multirow[t]{3}{*}{ HIP 25434} & 2010-02-17 & cc-1 & $4944 \pm 11$ & $154.5 \pm 0.1$ & $11.4 \pm 0.1$ & $\mathrm{~B}$ & \\
\hline & 2010-12-05 & cc-1 & $4937 \pm 5$ & $154.5 \pm 0.1$ & $12.0 \pm 0.1$ & $\mathrm{~B}$ & \\
\hline & 2012-11-21 & cc-1 & $4947 \pm 7$ & $155.1 \pm 0.1$ & $11.1 \pm 0.1$ & $\mathrm{~B}$ & \\
\hline \multirow[t]{2}{*}{ TYC 9162-0698-1 } & 2010-02-19 & 26 & & & & & Electronic table \\
\hline & 2011-01-24 & $26+33$ & & & & $\mathrm{~B}+\mathrm{U}$ & Electronic table \\
\hline \multirow[t]{4}{*}{ TYC 5346-132-1 } & 2009-11-23 & cc-1 & $6252 \pm 16$ & $1.8 \pm 0.2$ & $9.7 \pm 0.1$ & $\mathrm{~B}$ & \\
\hline & 2010-02-16 & cc-1 & $6260 \pm 9$ & $1.7 \pm 0.1$ & $9.8 \pm 0.1$ & $\mathrm{~B}$ & \\
\hline & 2009-11-23 & cc-2 & $6431 \pm 16$ & $0.2 \pm 0.2$ & $9.1 \pm 0.1$ & $\mathrm{~B}$ & \\
\hline & 2010-02-16 & cc-2 & $6434 \pm 9$ & $0.0 \pm 0.1$ & $9.1 \pm 0.1$ & B & \\
\hline HIP 30261 & $2009-11-23$ & none & & & & & \\
\hline \multirow[t]{2}{*}{ TYC 7617-0549-1 } & 2009-11-21 & cc-1 & $1848 \pm 16$ & $299.6 \pm 0.5$ & $12.6 \pm 0.1$ & $\mathrm{~B}$ & \\
\hline & 2012-11-22 & cc-1 & $1861 \pm 8$ & $298.6 \pm 0.3$ & $12.1 \pm 0.1$ & $\mathrm{~B}$ & \\
\hline TYC 9181-0466-1 & 2010-02-19 & none & & & & & New binary (see Table 5) \\
\hline \multirow[t]{2}{*}{ HIP 32235} & 2010-02-18 & cc-1 & $5559 \pm 12$ & $340.3 \pm 0.2$ & $11.8 \pm 0.1$ & $\mathrm{~B}$ & \\
\hline & 2010-12-30 & cc-1 & $5508 \pm 6$ & $339.8 \pm 0.1$ & $13.3 \pm 0.2$ & $\mathrm{~B}$ & \\
\hline \multirow[t]{8}{*}{ HIP 35564} & 2009-11-22 & cc-1 & $1865 \pm 20$ & $304.2 \pm 0.6$ & $15.3 \pm 0.3$ & $\mathrm{~B}$ & RV var \\
\hline & 2011-01-31 & cc-1 & $1852 \pm 8$ & $299.3 \pm 0.2$ & $14.4 \pm 0.7$ & $\mathrm{~B}$ & \\
\hline & 2009-11-22 & cc-2 & $3301 \pm 20$ & $148.9 \pm 0.3$ & $12.4 \pm 0.1$ & $\mathrm{~B}$ & \\
\hline & $2010-02-16$ & cc-2 & $3365 \pm 13$ & $148.7 \pm 0.2$ & $12.1 \pm 0.1$ & B & \\
\hline & 2011-01-31 & cc-2 & $3465 \pm 9$ & $149.8 \pm 0.2$ & $11.9 \pm 0.1$ & $\mathrm{~B}$ & \\
\hline & 2009-11-22 & $\mathrm{cc}-3$ & $6722 \pm 20$ & $1.5 \pm 0.2$ & $14.4 \pm 0.3$ & $\mathrm{~B}$ & \\
\hline & 2010-02-16 & cc-3 & $6660 \pm 12$ & $1.8 \pm 0.2$ & $14.0 \pm 0.4$ & B & \\
\hline & 2011-01-31 & cc-3 & $6581 \pm 8$ & $1.7 \pm 0.1$ & $13.7 \pm 0.4$ & $\mathrm{~B}$ & \\
\hline \multirow[t]{4}{*}{ TYC 8128-1946-1 } & 2009-11-21 & cc-1 & $5521 \pm 17$ & $178.2 \pm 0.2$ & $13.5 \pm 0.1$ & B & \\
\hline & 2011-01-20 & cc-1 & $5549 \pm 12$ & $178.1 \pm 0.2$ & $13.7 \pm 0.2$ & B & \\
\hline & 2009-11-21 & $\mathrm{cc}-2$ & $8211 \pm 17$ & $6.3 \pm 0.2$ & $9.4 \pm 0.1$ & $\mathrm{~B}$ & \\
\hline & 2011-01-20 & cc-2 & $8190 \pm 12$ & $6.3 \pm 0.1$ & $9.9 \pm 0.1$ & $\mathrm{~B}$ & \\
\hline \multirow[t]{3}{*}{ HIP 36414} & 2010-02-17 & cc-1 & $8296 \pm 21$ & $305.0 \pm 0.2$ & $12.8 \pm 0.7$ & B & $\mathrm{SB}, \mathrm{RV}$ var \\
\hline & 2011-01-31 & cc-1 & $8241 \pm 16$ & $304.5 \pm 0.1$ & $13.5 \pm 0.2$ & B & \\
\hline & $2010-02-17$ & cc-2 & $7076 \pm 19$ & $359.2 \pm 0.2$ & $13.6 \pm 0.2$ & $\mathrm{U}$ & \\
\hline \multirow[t]{5}{*}{ HIP 36948} & 2010-02-16 & cc-1 & $3485 \pm 21$ & $327.1 \pm 0.3$ & $14.1 \pm 0.2$ & B & The Moth ${ }^{a}$ \\
\hline & $2010-02-16$ & cc-2 & $6272 \pm 22$ & $315.5 \pm 0.2$ & $13.6 \pm 0.2$ & B & \\
\hline & $2010-02-16$ & cc-3 & $7217 \pm 20$ & $191.3 \pm 0.2$ & $13.3 \pm 0.4$ & B & \\
\hline & $2010-02-16$ & cc-4 & $8116 \pm 20$ & $171.1 \pm 0.2$ & $14.1 \pm 0.8$ & B & \\
\hline & $2010-02-16$ & cc-5 & $8206 \pm 20$ & $268.1 \pm 0.2$ & $10.1 \pm 0.1$ & B & \\
\hline HIP 37563 & 2010-02-18 & none & & & & & \\
\hline
\end{tabular}

Notes. Target name and observing date are given, as well as the different sources identified with their relative position, and relative flux, and their identification status based on follow-up observations. Sources are indicated follows: stationary background contaminants (B; based on a comoving companion probability $P_{\text {comoving } \chi^{2}}<1 \%$ and with a relative motion compatible with a background source); confirmed comoving companions (C; based on a stationary background contaminant probability $P_{\mathrm{BKG}, \chi^{2}}<1 \%$ and a relative motion compatible with a comoving companion); and undefined (U; when observed at only one epoch or when not satisfying the first two classifications). ${ }^{(a)}$ All background objects identified combining NaCo with HST data by Buenzli et al. (2010). ${ }^{(b)}$ Known binary (Brandner et al. 1996). ${ }^{(c)}$ Known binary (Köhler et al. 2000). ${ }^{(d)}$ Known binary (Chauvin et al. 2010). 
G. Chauvin et al.: The VLT/NaCo large program to probe the occurrence of exoplanets in wide orbits. II.

Table 6. continued.

\begin{tabular}{|c|c|c|c|c|c|c|c|}
\hline Name-1 & UT-Date & Nb Cand. & $\begin{array}{l}\text { Sep } \\
\text { (mas) }\end{array}$ & $\begin{array}{l}\text { PA } \\
(\mathrm{deg})\end{array}$ & $\begin{array}{l}\Delta H \\
(\mathrm{mag})\end{array}$ & Status & Comments \\
\hline \multirow[t]{11}{*}{ HIP 37923} & 2010-02-18 & cc-1 & $5439 \pm 10$ & $261.2 \pm 0.2$ & $12.6 \pm 0.1$ & $\mathrm{~B}$ & \\
\hline & 2011-01-01 & cc-1 & $5439 \pm 8$ & $259.8 \pm 0.1$ & $12.7 \pm 0.1$ & $\mathrm{~B}$ & \\
\hline & $2010-02-18$ & cc-2 & $5834 \pm 12$ & $55.5 \pm 0.1$ & $14.0 \pm 0.1$ & B & \\
\hline & 2011-01-01 & cc-2 & $5765 \pm 10$ & $56.5 \pm 0.1$ & $14.2 \pm 0.2$ & $\mathrm{~B}$ & \\
\hline & 2010-02-18 & cc-3 & $5997 \pm 12$ & $209.9 \pm 0.1$ & $12.4 \pm 0.1$ & $\mathrm{~B}$ & \\
\hline & 2011-01-01 & cc-3 & $6098 \pm 10$ & $209.1 \pm 0.1$ & $12.7 \pm 0.1$ & B & \\
\hline & 2010-02-18 & cc-4 & $7070 \pm 12$ & $28.2 \pm 0.1$ & $14.1 \pm 0.3$ & $\mathrm{~B}$ & \\
\hline & 2011-01-01 & cc-4 & $6947 \pm 10$ & $28.8 \pm 0.1$ & $14.4 \pm 0.2$ & B & \\
\hline & 2010-02-18 & cc-5 & $8076 \pm 13$ & $65.1 \pm 0.1$ & $9.1 \pm 0.1$ & B & \\
\hline & 2011-01-01 & cc-5 & $8029 \pm 10$ & $65.9 \pm 0.1$ & $9.6 \pm 0.1$ & B & \\
\hline & 2010-02-18 & cc-6 & $8677 \pm 15$ & $43.1 \pm 0.1$ & $13.8 \pm 0.2$ & $\mathrm{U}$ & \\
\hline \multirow[t]{2}{*}{ TYC 8927-3620-1 } & 2010-02-19 & none & & & & & New binary (see Table 5) \\
\hline & & & & & & & $\begin{array}{l}\text { Third component at } \sim 4.8^{\prime \prime} \\
\text { resolved by } 2 \text { MASS }\end{array}$ \\
\hline HIP 46634 & 2009-11-24 & none & & & & & \\
\hline HIP 47646 & 2010-02-18 & none & & & & & \\
\hline \multirow[t]{27}{*}{ TWA-21 } & 2010-02-18 & cc-1 & $2353 \pm 11$ & $30.9 \pm 0.3$ & $13.8 \pm 0.3$ & B & \\
\hline & 2011-01-13 & cc-1 & $2339 \pm 7$ & $31.5 \pm 0.2$ & $14.2 \pm 0.4$ & B & \\
\hline & 2010-02-18 & cc-2 & $2508 \pm 11$ & $342.2 \pm 0.3$ & $13.9 \pm 0.3$ & B & \\
\hline & 2011-01-13 & cc-2 & $2489 \pm 7$ & $342.7 \pm 0.2$ & $14.2 \pm 0.3$ & B & \\
\hline & 2010-02-18 & cc-3 & $3152 \pm 11$ & $67.6 \pm 0.2$ & $12.2 \pm 0.1$ & B & \\
\hline & 2011-01-13 & cc-3 & $3178 \pm 7$ & $68.1 \pm 0.2$ & $12.8 \pm 0.1$ & B & \\
\hline & 2010-02-18 & cc-4 & $4968 \pm 11$ & $94.0 \pm 0.2$ & $13.4 \pm 0.2$ & B & \\
\hline & 2011-01-13 & cc-4 & $5003 \pm 6$ & $94.1 \pm 0.1$ & $14.0 \pm 0.3$ & B & \\
\hline & $2010-02-18$ & cc-5 & $5231 \pm 12$ & $241.6 \pm 0.2$ & $8.9 \pm 0.1$ & B & \\
\hline & 2011-01-13 & cc-5 & $5210 \pm 8$ & $241.2 \pm 0.1$ & $9.4 \pm 0.1$ & B & \\
\hline & $2010-02-18$ & cc-6 & $5355 \pm 12$ & $71.1 \pm 0.2$ & $11.7 \pm 0.1$ & B & \\
\hline & 2011-01-13 & cc-6 & $5387 \pm 7$ & $71.3 \pm 0.1$ & $12.4 \pm 0.1$ & B & \\
\hline & $2010-02-18$ & cc-7 & $5602 \pm 12$ & $24.6 \pm 0.1$ & $9.1 \pm 0.1$ & B & \\
\hline & 2011-01-13 & cc-7 & $5599 \pm 8$ & $25.0 \pm 0.1$ & $9.7 \pm 0.1$ & B & \\
\hline & 2010-02-18 & cc-8 & $5712 \pm 12$ & $27.8 \pm 0.1$ & $12.7 \pm 0.1$ & B & \\
\hline & 2011-01-13 & cc-8 & $5717 \pm 9$ & $28.0 \pm 0.1$ & $13.2 \pm 0.1$ & B & \\
\hline & 2010-02-18 & cc-9 & $5801 \pm 12$ & $245.6 \pm 0.1$ & $10.2 \pm 0.1$ & B & \\
\hline & 2011-01-13 & cc-9 & $5776 \pm 8$ & $245.3 \pm 0.1$ & $10.7 \pm 0.1$ & B & \\
\hline & 2010-02-18 & cc-10 & $5833 \pm 11$ & $102.6 \pm 0.2$ & $13.4 \pm 0.2$ & B & \\
\hline & 2011-01-13 & cc- 10 & $5879 \pm 7$ & $102.6 \pm 0.1$ & $14.0 \pm 0.2$ & B & \\
\hline & 2010-02-18 & cc-11 & $6097 \pm 12$ & $107.7 \pm 0.1$ & $12.6 \pm 0.1$ & B & \\
\hline & 2011-01-13 & cc-11 & $6138 \pm 7$ & $107.7 \pm 0.1$ & $13.3 \pm 0.1$ & B & \\
\hline & 2010-02-18 & cc-12 & $6951 \pm 13$ & $147.5 \pm 0.1$ & $13.6 \pm 0.2$ & B & \\
\hline & 2011-01-13 & cc-12 & $6990 \pm 10$ & $147.4 \pm 0.1$ & $14.4 \pm 0.4$ & B & \\
\hline & $2010-02-18$ & cc-13 & $3369 \pm 11$ & $165.3 \pm 0.2$ & $14.3 \pm 0.3$ & $\mathrm{U}$ & \\
\hline & 2010-02-18 & cc-14 & $5948 \pm 13$ & $228.5 \pm 0.1$ & $15.0 \pm 0.4$ & $\mathrm{U}$ & \\
\hline & 2011-01-13 & cc-15 & $7049 \pm 7$ & $9.4 \pm 0.1$ & $12.9 \pm 0.1$ & $\mathrm{U}$ & \\
\hline \multirow[t]{10}{*}{ TYC 7188-0575-1 } & $2010-02-16$ & cc-1 & $4238 \pm 15$ & $296.5 \pm 0.2$ & $15.2 \pm 0.4$ & $\mathrm{~B}$ & SB2 \\
\hline & 2011-01-27 & cc-1 & $4148 \pm 12$ & $297.5 \pm 0.2$ & $14.8 \pm 0.4$ & $\mathrm{~B}$ & \\
\hline & $2010-02-16$ & cc-2 & $4741 \pm 15$ & $61.7 \pm 0.2$ & $11.6 \pm 0.1$ & $\mathrm{~B}$ & \\
\hline & 2011-01-27 & cc-2 & $4843 \pm 12$ & $62.2 \pm 0.2$ & $11.4 \pm 0.1$ & B & \\
\hline & 2010-02-16 & cc-3 & $5329 \pm 15$ & $186.0 \pm 0.2$ & $11.9 \pm 0.1$ & $\mathrm{~B}$ & \\
\hline & 2011-01-27 & cc-3 & $5303 \pm 11$ & $185.1 \pm 0.2$ & $11.8 \pm 0.1$ & $\mathrm{~B}$ & \\
\hline & 2010-02-16 & cc-4 & $7391 \pm 15$ & $279.5 \pm 0.2$ & $13.2 \pm 0.2$ & $\mathrm{~B}$ & \\
\hline & 2011-01-27 & cc-4 & $7282 \pm 11$ & $279.8 \pm 0.1$ & $13.1 \pm 0.2$ & $\mathrm{~B}$ & \\
\hline & $2010-02-16$ & cc-5 & $8020 \pm 15$ & $5.1 \pm 0.2$ & $11.6 \pm 0.1$ & $\mathrm{~B}$ & \\
\hline & 2011-01-27 & cc-5 & $8058 \pm 11$ & $5.8 \pm 0.1$ & $11.7 \pm 0.1$ & $\mathrm{~B}$ & \\
\hline TYC 6069-1214-1 & $2010-02-17$ & none & & & & & \\
\hline \multirow[t]{9}{*}{ TYC 7722-0207-1 } & 2010-02-17 & cc-1 & $3981 \pm 11$ & $33.5 \pm 0.2$ & $11.7 \pm 0.1$ & B & \\
\hline & 2011-01-29 & cc-1 & $3978 \pm 6$ & $35.0 \pm 0.1$ & $11.5 \pm 0.1$ & $\mathrm{~B}$ & \\
\hline & 2010-02-17 & cc-2 & $4369 \pm 12$ & $228.1 \pm 0.2$ & $7.3 \pm 0.1$ & B & \\
\hline & 2011-01-29 & cc-2 & $4355 \pm 7$ & $226.9 \pm 0.1$ & $7.1 \pm 0.1$ & $\mathrm{~B}$ & \\
\hline & 2010-02-17 & cc-3 & $8516 \pm 11$ & $105.2 \pm 0.1$ & $9.4 \pm 0.1$ & B & \\
\hline & 2011-01-29 & cc-3 & $8602 \pm 6$ & $105.4 \pm 0.1$ & $9.1 \pm 0.1$ & B & \\
\hline & 2010-02-17 & cc-4 & $1742 \pm 10$ & $329.8 \pm 0.3$ & $13.9 \pm 0.5$ & $\mathrm{U}$ & \\
\hline & 2011-01-29 & cc-5 & $7958 \pm 11$ & $317.3 \pm 0.1$ & $10.1 \pm 0.1$ & $\mathrm{U}$ & \\
\hline & 2011-01-29 & cc-6 & $7988 \pm 11$ & $42.2 \pm 0.1$ & $12.4 \pm 0.1$ & $\mathrm{U}$ & \\
\hline
\end{tabular}


Table 6. continued.

\begin{tabular}{|c|c|c|c|c|c|c|c|}
\hline Name-1 & UT-Date & Nb Cand. & $\begin{array}{l}\text { Sep } \\
\text { (mas) }\end{array}$ & $\begin{array}{l}\text { PA } \\
\text { (deg) }\end{array}$ & $\begin{array}{l}\Delta H \\
(\mathrm{mag})\end{array}$ & Status & Comments \\
\hline TYC 7743-1091-1 & $2010-02-19$ & none & & & & & \\
\hline \multirow[t]{2}{*}{ HIP 58240} & 2010-02-16 & cc-1 & $5761 \pm 21$ & $179.6 \pm 0.2$ & $13.0 \pm 0.2$ & $\mathrm{~B}$ & \\
\hline & 2011-01-29 & cc-1 & $5770 \pm 5$ & $178.1 \pm 0.1$ & $12.9 \pm 0.1$ & $\mathrm{~B}$ & \\
\hline TYC 9231-1566-1 & $2010-02-19$ & none & & & & & New binary (see Table 5) \\
\hline \multirow[t]{2}{*}{ TYC 8979-1683-1 } & $2010-02-18$ & $54(+16)$ & & & & & Electronic table \\
\hline & 2011-05-11 & 54 & & & & $\mathrm{~B}(+\mathrm{U})$ & Electronic table \\
\hline \multirow[t]{2}{*}{ TYC 8989-0583-1 } & $2010-02-18$ & none & & & & & New binary (see Table 5) \\
\hline & 2010-06-16 & none & & & & & \\
\hline \multirow{15}{*}{ TYC 9245-0617-1 } & 2010-02-18 & cc-1 & $3771 \pm 10$ & $32.2 \pm 0.2$ & $9.5 \pm 0.1$ & $\mathrm{~B}$ & \\
\hline & $2013-02-11$ & $\mathrm{cc}-1$ & $3860 \pm 5$ & $33.2 \pm 0.1$ & $9.1 \pm 0.1$ & B & \\
\hline & 2010-02-18 & $\mathrm{cc}-2$ & $3942 \pm 10$ & $119.4 \pm 0.2$ & $5.9 \pm 0.1$ & B & \\
\hline & 2011-04-04 & cc-2 & $3974 \pm 10$ & $118.9 \pm 0.2$ & $6.0 \pm 0.1$ & $\mathrm{~B}$ & \\
\hline & $2013-02-11$ & $\mathrm{cc}-2$ & $4015 \pm 5$ & $118.4 \pm 0.1$ & $5.8 \pm 0.1$ & B & \\
\hline & $2010-02-18$ & cc-3 & $6544 \pm 9$ & $183.4 \pm 0.1$ & $10.9 \pm 0.1$ & $\mathrm{~B}$ & \\
\hline & $2013-02-11$ & cc-3 & $6517 \pm 3$ & $182.4 \pm 0.1$ & $10.9 \pm 0.3$ & $\mathrm{~B}$ & \\
\hline & $2010-02-18$ & cc-4 & $7448 \pm 11$ & $241.0 \pm 0.1$ & $10.2 \pm 0.1$ & $\mathrm{~B}$ & \\
\hline & 2013-02-11 & cc-4 & $7342 \pm 8$ & $241.0 \pm 0.1$ & $10.2 \pm 0.2$ & B & \\
\hline & $2010-02-18$ & cc-5 & $4590 \pm 10$ & $306.1 \pm 0.1$ & $12.8 \pm 0.1$ & $\mathrm{U}$ & \\
\hline & $2010-02-18$ & cc-6 & $5603 \pm 9$ & $263.3 \pm 0.1$ & $14.5 \pm 0.3$ & $\mathrm{U}$ & \\
\hline & 2010-02-18 & cc-7 & $5887 \pm 9$ & $179.7 \pm 0.1$ & $12.2 \pm 0.1$ & $\mathrm{U}$ & \\
\hline & $2010-02-18$ & cc-8 & $6149 \pm 10$ & $251.4 \pm 0.1$ & $13.3 \pm 0.2$ & $\mathrm{U}$ & \\
\hline & $2010-02-18$ & cc-9 & $7432 \pm 12$ & $146.0 \pm 0.1$ & $11.9 \pm 0.1$ & $\mathrm{U}$ & \\
\hline & $2010-02-18$ & cc-10 & $8529 \pm 10$ & $73.1 \pm 0.1$ & $12.9 \pm 0.2$ & $\mathrm{U}$ & \\
\hline \multirow[t]{6}{*}{ HIP 63862} & $2010-02-18$ & cc-1 & $4231 \pm 16$ & $28.0 \pm 0.2$ & $12.9 \pm 0.1$ & B & \\
\hline & 2011-07-02 & $\mathrm{cc}-1$ & $4315 \pm 5$ & $30.7 \pm 0.1$ & $13.1 \pm 0.1$ & B & \\
\hline & $2010-02-18$ & cc-2 & $5536 \pm 17$ & $206.7 \pm 0.2$ & $12.8 \pm 0.1$ & B & \\
\hline & 2011-07-02 & cc-2 & $5478 \pm 5$ & $204.8 \pm 0.1$ & $13.3 \pm 0.1$ & B & \\
\hline & 2011-07-02 & cc-3 & $7186 \pm 5$ & $160.1 \pm 0.1$ & $14.1 \pm 0.2$ & $\mathrm{U}$ & \\
\hline & 2011-07-02 & $\mathrm{cc}-4$ & $8169 \pm 10$ & $142.8 \pm 0.1$ & $12.7 \pm 0.1$ & $\mathrm{U}$ & \\
\hline \multirow{16}{*}{ TYC 7796-2110-1 } & $2010-02-16$ & cc-1 & $3264 \pm 15$ & $127.0 \pm 0.3$ & $11.3 \pm 0.1$ & $\mathrm{~B}$ & \\
\hline & 2011-05-11 & $\mathrm{cc}-1$ & $3306 \pm 4$ & $126.3 \pm 0.1$ & $11.1 \pm 0.3$ & B & \\
\hline & 2013-03-22 & cc-1 & $3314 \pm 9$ & $125.2 \pm 0.2$ & $11.2 \pm 0.1$ & B & \\
\hline & $2010-02-16$ & cc-2 & $3990 \pm 16$ & $141.6 \pm 0.2$ & $8.7 \pm 0.1$ & B & \\
\hline & 2011-05-11 & cc-2 & $4018 \pm 5$ & $141.1 \pm 0.1$ & $8.8 \pm 0.1$ & $\mathrm{~B}$ & \\
\hline & $2013-03-22$ & cc-2 & $4034 \pm 9$ & $140.3 \pm 0.1$ & $8.7 \pm 0.1$ & B & \\
\hline & $2010-02-16$ & cc-3 & $4111 \pm 15$ & $109.7 \pm 0.2$ & $13.5 \pm 0.2$ & B & \\
\hline & $2013-03-22$ & cc-3 & $4190 \pm 8$ & $108.5 \pm 0.1$ & $13.6 \pm 0.3$ & B & \\
\hline & $2010-02-16$ & cc-4 & $5231 \pm 16$ & $151.6 \pm 0.2$ & $13.2 \pm 0.1$ & B & \\
\hline & $2013-03-22$ & cc-4 & $5194 \pm 10$ & $150.7 \pm 0.1$ & $13.3 \pm 0.2$ & $\mathrm{~B}$ & \\
\hline & $2010-02-16$ & cc-5 & $6324 \pm 17$ & $214.1 \pm 0.2$ & $12.5 \pm 0.1$ & $\mathrm{~B}$ & \\
\hline & 2013-03-22 & $\mathrm{cc}-5$ & $6211 \pm 11$ & $214.0 \pm 0.1$ & $12.4 \pm 0.1$ & $\mathrm{~B}$ & \\
\hline & $2010-02-16$ & cc-6 & $7111 \pm 16$ & $62.6 \pm 0.2$ & $10.8 \pm 0.1$ & B & \\
\hline & 2011-05-11 & cc-6 & $7145 \pm 7$ & $62.8 \pm 0.1$ & $11.1 \pm 0.3$ & $\mathrm{~B}$ & \\
\hline & $2013-03-22$ & cc-6 & $7240 \pm 10$ & $62.8 \pm 0.1$ & $10.8 \pm 0.1$ & $\mathrm{~B}$ & \\
\hline & $2010-02-16$ & $\mathrm{cc}-7$ & $8743 \pm 18$ & $48.5 \pm 0.1$ & $14.0 \pm 0.4$ & $\mathrm{U}$ & \\
\hline TYC 9010-1272-1 & $2010-02-18$ & none & & & & & New binary (see Table 5) \\
\hline \multirow[t]{7}{*}{ HIP 70351} & $2010-02-17$ & $\mathrm{cc}-1$ & $2971 \pm 10$ & $258.4 \pm 0.2$ & $14.7 \pm 0.4$ & $\mathrm{U}$ & \\
\hline & $2010-02-17$ & $\mathrm{cc}-2$ & $4664 \pm 11$ & $288.7 \pm 0.2$ & $11.4 \pm 0.1$ & $\mathrm{U}$ & \\
\hline & $2010-02-17$ & cc-3 & $4973 \pm 10$ & $4.0 \pm 0.2$ & $13.9 \pm 0.2$ & $\mathrm{U}$ & \\
\hline & $2010-02-17$ & cc-4 & $6572 \pm 10$ & $262.2 \pm 0.1$ & $13.5 \pm 0.1$ & $\mathrm{U}$ & \\
\hline & $2010-02-17$ & cc-5 & $6615 \pm 13$ & $235.8 \pm 0.1$ & $13.0 \pm 0.1$ & $\mathrm{U}$ & \\
\hline & $2010-02-17$ & cc-6 & $6820 \pm 13$ & $145.7 \pm 0.1$ & $11.0 \pm 0.1$ & $\mathrm{U}$ & \\
\hline & $2010-02-17$ & cc-7 & $7266 \pm 12$ & $118.2 \pm 0.1$ & $12.9 \pm 0.1$ & $\mathrm{U}$ & \\
\hline & $2010-02-16$ & $\mathrm{cc}-1$ & $6404 \pm 16$ & $28.4 \pm 0.2$ & $16.3 \pm 0.4$ & $\mathrm{U}$ & \\
\hline \multirow[t]{5}{*}{ HIP 71933} & $2010-06-15$ & $\mathrm{cc}-1$ & $4934 \pm 9$ & $12.6 \pm 0.1$ & $10.0 \pm 0.1$ & $\mathrm{~B}$ & \\
\hline & 2011-04-06 & $\mathrm{cc}-1$ & $4952 \pm 2$ & $12.8 \pm 0.1$ & $9.4 \pm 0.1$ & B & \\
\hline & $2013-02-23$ & cc-1 & $5031 \pm 3$ & $13.1 \pm 0.1$ & $10.0 \pm 0.1$ & $\mathrm{~B}$ & \\
\hline & $2010-06-15$ & $\mathrm{cc}-2$ & $5864 \pm 11$ & $54.1 \pm 0.1$ & $11.3 \pm 0.1$ & $\mathrm{~B}$ & \\
\hline & $2013-02-23$ & $\mathrm{cc}-2$ & $5958 \pm 7$ & $54.0 \pm 0.1$ & $11.3 \pm 0.1$ & $\mathrm{~B}$ & \\
\hline
\end{tabular}


G. Chauvin et al.: The VLT/NaCo large program to probe the occurrence of exoplanets in wide orbits. II.

Table 6. continued.

\begin{tabular}{|c|c|c|c|c|c|c|c|}
\hline Name-1 & UT-Date & Nb Cand. & $\begin{array}{l}\text { Sep } \\
\text { (mas) }\end{array}$ & $\begin{array}{l}\text { PA } \\
\text { (deg) }\end{array}$ & $\begin{array}{l}\Delta H \\
(\mathrm{mag})\end{array}$ & Status & Comments \\
\hline \multirow[t]{10}{*}{ HIP 71933} & $2010-06-15$ & cc-3 & $7987 \pm 9$ & $12.5 \pm 0.1$ & $10.4 \pm 0.1$ & B & \\
\hline & 2011-04-06 & cc-3 & $8006 \pm 3$ & $12.8 \pm 0.1$ & $9.8 \pm 0.2$ & $\mathrm{~B}$ & \\
\hline & $2013-02-23$ & cc-3 & $8096 \pm 4$ & $13.1 \pm 0.1$ & $10.4 \pm 0.1$ & $\mathrm{~B}$ & \\
\hline & $2010-06-15$ & cc-4 & $9258 \pm 12$ & $205.1 \pm 0.1$ & $9.2 \pm 0.1$ & B & \\
\hline & $2013-02-23$ & cc-4 & $9127 \pm 8$ & $205.1 \pm 0.1$ & $9.5 \pm 0.1$ & $\mathrm{~B}$ & \\
\hline & $2010-06-15$ & cc-5 & $5434 \pm 10$ & $22.6 \pm 0.1$ & $13.3 \pm 0.1$ & $\mathrm{U}$ & \\
\hline & $2010-06-15$ & cc-6 & $6963 \pm 10$ & $107.8 \pm 0.1$ & $13.9 \pm 0.2$ & $\mathrm{U}$ & \\
\hline & $2010-06-15$ & cc-7 & $7158 \pm 12$ & $130.1 \pm 0.1$ & $12.4 \pm 0.1$ & $\mathrm{U}$ & \\
\hline & $2010-06-15$ & cc-8 & $7968 \pm 11$ & $202.0 \pm 0.1$ & $13.9 \pm 0.3$ & $\mathrm{U}$ & \\
\hline & $2010-06-15$ & cc-9 & $8756 \pm 9$ & $12.5 \pm 0.1$ & $12.9 \pm 0.2$ & $\mathrm{U}$ & \\
\hline HIP 72399 & 2011-05-27 & none & & & & & SB1, RV var \\
\hline TYC 7835-2569-1 & 2011-05-11 & none & & & & & $\mathrm{SB} 2+$ Known binary $^{b}$ \\
\hline \multirow[t]{8}{*}{ HIP 76829} & $2010-06-15$ & $\mathrm{cc}-1$ & $2393 \pm 4$ & $46.8 \pm 0.1$ & $15.0 \pm 0.4$ & $\mathrm{~B}$ & \\
\hline & 2011-06-27 & cc-1 & $2705 \pm 9$ & $45.7 \pm 0.2$ & $14.5 \pm 0.5$ & $\mathrm{~B}$ & \\
\hline & $2010-06-15$ & cc-2 & $6368 \pm 6$ & $111.7 \pm 0.1$ & $14.8 \pm 0.3$ & $\mathrm{~B}$ & \\
\hline & 2011-06-27 & cc-2 & $6434 \pm 10$ & $109.1 \pm 0.1$ & $14.2 \pm 0.3$ & B & \\
\hline & $2010-06-15$ & cc-3 & $4481 \pm 6$ & $310.5 \pm 0.1$ & $16.3 \pm 0.6$ & $\mathrm{U}$ & \\
\hline & $2010-06-15$ & cc-4 & $5272 \pm 3$ & $272.4 \pm 0.1$ & $16.3 \pm 0.4$ & $\mathrm{U}$ & \\
\hline & $2010-06-15$ & cc-5 & $5900 \pm 3$ & $91.3 \pm 0.1$ & $16.2 \pm 0.4$ & $\mathrm{U}$ & \\
\hline & 2010-06-15 & cc-6 & $8618 \pm 11$ & $142.8 \pm 0.1$ & $15.2 \pm 0.4$ & $\mathrm{U}$ & \\
\hline TYC 6781-0415-1 & 2011-07-20 & none & & & & & \\
\hline TYC 6786-0811-1 & $2010-07-29$ & none & & & & & Known binary ${ }^{c}$ \\
\hline \multirow[t]{10}{*}{ HIP 78747} & 2010-07-29 & cc-1 & $3892 \pm 2$ & $0.6 \pm 0.1$ & $11.9 \pm 0.1$ & B & \\
\hline & 2011-06-28 & cc-1 & $3990 \pm 4$ & $2.2 \pm 0.1$ & $12.0 \pm 0.1$ & $\mathrm{~B}$ & \\
\hline & $2010-07-29$ & cc-2 & $6154 \pm 5$ & $292.2 \pm 0.1$ & $12.8 \pm 0.1$ & $\mathrm{~B}$ & \\
\hline & 2011-06-28 & cc-2 & $6110 \pm 6$ & $293.5 \pm 0.1$ & $13.0 \pm 0.2$ & $\mathrm{~B}$ & \\
\hline & $2010-07-29$ & cc-3 & $6633 \pm 8$ & $53.9 \pm 0.1$ & $12.7 \pm 0.1$ & $\mathrm{~B}$ & \\
\hline & 2011-06-28 & cc-3 & $6788 \pm 9$ & $53.9 \pm 0.1$ & $12.8 \pm 0.2$ & $\mathrm{~B}$ & \\
\hline & $2010-07-29$ & cc-4 & $5508 \pm 6$ & $59.0 \pm 0.1$ & $14.6 \pm 0.2$ & $\mathrm{U}$ & \\
\hline & 2010-07-29 & cc-5 & $5949 \pm 2$ & $1.1 \pm 0.1$ & $14.7 \pm 0.3$ & $\mathrm{U}$ & \\
\hline & $2010-07-29$ & cc-6 & $7557 \pm 5$ & $200.8 \pm 0.1$ & $14.0 \pm 0.2$ & $\mathrm{U}$ & \\
\hline & 2011-06-28 & cc-7 & $7419 \pm 5$ & $255.3 \pm 0.1$ & $13.2 \pm 0.2$ & $\mathrm{U}$ & \\
\hline TYC 6209-0769-1 & 2011-08-19 & cc-1 & $5473 \pm 3$ & $198.6 \pm 0.1$ & $8.2 \pm 0.1$ & $\mathrm{U}$ & \\
\hline \multirow[t]{7}{*}{ HIP 79958} & 2011-06-27 & cc-1 & $3583 \pm 4$ & $29.3 \pm 0.1$ & $11.7 \pm 0.3$ & $\mathrm{U}$ & \\
\hline & 2011-06-27 & cc-2 & $3689 \pm 1$ & $88.8 \pm 0.1$ & $9.4 \pm 0.1$ & $\mathrm{U}$ & \\
\hline & 2011-06-27 & cc-3 & $4120 \pm 2$ & $281.1 \pm 0.1$ & $10.2 \pm 0.1$ & $\mathrm{U}$ & \\
\hline & 2011-06-27 & cc-4 & $4633 \pm 4$ & $151.3 \pm 0.1$ & $11.5 \pm 0.2$ & $\mathrm{U}$ & \\
\hline & 2011-06-27 & cc-5 & $5986 \pm 2$ & $169.4 \pm 0.1$ & $10.9 \pm 0.1$ & $\mathrm{U}$ & \\
\hline & 2011-06-27 & cc-6 & $6195 \pm 1$ & $177.4 \pm 0.1$ & $10.6 \pm 0.1$ & $\mathrm{U}$ & \\
\hline & 2011-06-27 & cc-7 & $7628 \pm 2$ & $350.9 \pm 0.1$ & $11.3 \pm 0.3$ & $\mathrm{U}$ & \\
\hline \multirow[t]{10}{*}{ HIP 80290} & 2011-08-08 & cc-1 & $2688 \pm 1$ & $184.6 \pm 0.1$ & $8.5 \pm 0.1$ & $\mathrm{~B}$ & \\
\hline & $2012-08-12$ & cc-1 & $2665 \pm 7$ & $184.3 \pm 0.2$ & $9.1 \pm 0.1$ & B & \\
\hline & 2011-08-08 & cc-2 & $3340 \pm 1$ & $257.5 \pm 0.1$ & $1.9 \pm 0.1$ & $\mathrm{C}$ & \\
\hline & 2012-08-12 & cc-2 & $3335 \pm 8$ & $257.6 \pm 0.2$ & $2.6 \pm 0.1$ & $\mathrm{C}$ & New binary (see Table 5) \\
\hline & 2011-08-08 & cc-3 & $7425 \pm 9$ & $143.6 \pm 0.1$ & $6.9 \pm 0.1$ & $\mathrm{~B}$ & \\
\hline & 2012-08-12 & cc-3 & $7417 \pm 12$ & $143.4 \pm 0.1$ & $7.6 \pm 0.1$ & $\mathrm{~B}$ & \\
\hline & $2012-08-12$ & cc-4 & $2097 \pm 8$ & $34.3 \pm 0.2$ & $12.4 \pm 0.3$ & $\mathrm{U}$ & \\
\hline & $2012-08-12$ & cc-5 & $2245 \pm 8$ & $291.3 \pm 0.2$ & $11.1 \pm 0.1$ & $\mathrm{U}$ & \\
\hline & $2012-08-12$ & cc-6 & $6186 \pm 8$ & $94.6 \pm 0.1$ & $12.0 \pm 0.1$ & $\mathrm{U}$ & \\
\hline & $2012-08-12$ & cc-7 & $8629 \pm 11$ & $298.8 \pm 0.1$ & $11.0 \pm 0.1$ & $\mathrm{U}$ & \\
\hline \multirow[t]{12}{*}{ HIP 80758} & $2010-07-29$ & cc-1 & $2210 \pm 3$ & $163.6 \pm 0.1$ & $12.9 \pm 0.1$ & $\mathrm{~B}$ & \\
\hline & 2011-05-11 & cc-1 & $2171 \pm 7$ & $163.3 \pm 0.2$ & $12.2 \pm 0.2$ & $\mathrm{~B}$ & \\
\hline & 2010-07-29 & cc-2 & $2221 \pm 4$ & $241.1 \pm 0.1$ & $12.5 \pm 0.1$ & $\mathrm{~B}$ & \\
\hline & 2011-05-11 & cc-2 & $2192 \pm 7$ & $242.1 \pm 0.2$ & $11.8 \pm 0.2$ & B & \\
\hline & $2010-07-29$ & cc-3 & $2413 \pm 4$ & $321.1 \pm 0.1$ & $12.8 \pm 0.1$ & B & \\
\hline & 2011-05-11 & cc-3 & $2455 \pm 7$ & $321.7 \pm 0.2$ & $12.3 \pm 0.2$ & $\mathrm{~B}$ & \\
\hline & $2010-07-29$ & cc-4 & $4686 \pm 6$ & $236.1 \pm 0.1$ & $11.5 \pm 0.1$ & B & \\
\hline & 2011-05-11 & cc-4 & $4651 \pm 8$ & $236.6 \pm 0.1$ & $10.9 \pm 0.1$ & B & \\
\hline & $2010-07-29$ & cc-5 & $5228 \pm 7$ & $304.1 \pm 0.1$ & $12.7 \pm 0.1$ & B & \\
\hline & 2011-05-11 & cc-5 & $5256 \pm 9$ & $304.6 \pm 0.1$ & $12.1 \pm 0.1$ & B & \\
\hline & $2010-07-29$ & cc-6 & $5229 \pm 5$ & $70.3 \pm 0.1$ & $13.9 \pm 0.2$ & B & \\
\hline & 2011-05-11 & cc-6 & $5215 \pm 8$ & $69.9 \pm 0.1$ & $12.9 \pm 0.2$ & B & \\
\hline
\end{tabular}


Table 6. continued.

\begin{tabular}{|c|c|c|c|c|c|c|c|}
\hline Name-1 & UT-Date & Nb Cand. & $\begin{array}{l}\text { Sep } \\
\text { (mas) }\end{array}$ & $\begin{array}{l}\text { PA } \\
\text { (deg) }\end{array}$ & $\begin{array}{l}\Delta H \\
(\mathrm{mag})\end{array}$ & Status & Comments \\
\hline \multirow[t]{9}{*}{ HIP 80758} & $2010-07-29$ & cc-7 & $5441 \pm 5$ & $108.0 \pm 0.1$ & $11.9 \pm 0.1$ & $\mathrm{~B}$ & \\
\hline & 2011-05-11 & $\mathrm{cc}-7$ & $5418 \pm 7$ & $107.7 \pm 0.1$ & $10.9 \pm 0.1$ & B & \\
\hline & $2010-07-29$ & cc- 8 & $5489 \pm 6$ & $25.2 \pm 0.1$ & $10.2 \pm 0.1$ & B & \\
\hline & 2011-05-11 & cc- 8 & $5523 \pm 8$ & $24.8 \pm 0.1$ & $9.6 \pm 0.1$ & $\mathrm{~B}$ & \\
\hline & $2010-07-29$ & cc-9 & $7495 \pm 4$ & $79.1 \pm 0.1$ & $6.7 \pm 0.1$ & $\mathrm{~B}$ & \\
\hline & 2011-05-11 & cc-9 & $7472 \pm 7$ & $78.7 \pm 0.1$ & $5.7 \pm 0.1$ & $\mathrm{~B}$ & \\
\hline & $2010-07-29$ & cc-10 & $7925 \pm 3$ & $265.4 \pm 0.1$ & $12.7 \pm 0.2$ & $\mathrm{~B}$ & \\
\hline & 2011-05-11 & cc-10 & $7897 \pm 7$ & $265.5 \pm 0.1$ & $12.4 \pm 0.3$ & $\mathrm{~B}$ & \\
\hline & $2010-07-29$ & cc-11 & $3005 \pm 4$ & $22.0 \pm 0.1$ & $14.4 \pm 0.3$ & $\mathrm{U}$ & \\
\hline \multirow[t]{3}{*}{ TYC 6818-1336-1 } & 2011-07-20 & cc-1 & $3382 \pm 10$ & $302.7 \pm 0.2$ & $7.3 \pm 0.1$ & $\mathrm{U}$ & \\
\hline & 2011-07-20 & cc-2 & $5824 \pm 10$ & $291.7 \pm 0.1$ & $5.5 \pm 0.1$ & $\mathrm{U}$ & \\
\hline & 2011-07-20 & cc-3 & $8914 \pm 14$ & $52.1 \pm 0.1$ & $2.8 \pm 0.1$ & $\mathrm{U}$ & \\
\hline TYC 6815-0084-1 & 2013-06-02 & none & & & & & SB2? \\
\hline \multirow{11}{*}{ TYC 6815-0874-1 } & $2012-08-13$ & $\mathrm{cc}-1$ & $2094 \pm 16$ & $229.6 \pm 0.4$ & $12.2 \pm 0.2$ & $\mathrm{U}$ & \\
\hline & $2012-08-13$ & cc-2 & $2224 \pm 16$ & $333.4 \pm 0.4$ & $11.9 \pm 0.2$ & $\mathrm{U}$ & \\
\hline & $2012-08-13$ & cc-3 & $2713 \pm 16$ & $280.7 \pm 0.4$ & $12.8 \pm 0.2$ & $\mathrm{U}$ & \\
\hline & $2012-08-13$ & cc-4 & $2754 \pm 16$ & $12.8 \pm 0.3$ & $11.1 \pm 0.1$ & $\mathrm{U}$ & \\
\hline & $2012-08-13$ & cc-5 & $3801 \pm 16$ & $171.0 \pm 0.3$ & $9.6 \pm 0.1$ & $\mathrm{U}$ & \\
\hline & $2012-08-13$ & cc-6 & $4035 \pm 17$ & $36.9 \pm 0.2$ & $12.1 \pm 0.1$ & $\mathrm{U}$ & \\
\hline & $2012-08-13$ & cc-7 & $4940 \pm 17$ & $164.2 \pm 0.2$ & $13.2 \pm 0.2$ & $\mathrm{U}$ & \\
\hline & $2012-08-13$ & cc- 8 & $6046 \pm 17$ & $285.5 \pm 0.2$ & $12.9 \pm 0.2$ & $\mathrm{U}$ & \\
\hline & $2012-08-13$ & cc-9 & $6569 \pm 17$ & $22.6 \pm 0.2$ & $13.1 \pm 0.2$ & $\mathrm{U}$ & \\
\hline & $2012-08-13$ & cc-10 & $8354 \pm 19$ & $306.1 \pm 0.1$ & $12.4 \pm 0.2$ & $\mathrm{U}$ & \\
\hline & $2012-08-13$ & $\mathrm{cc}-11$ & $9053 \pm 19$ & $125.0 \pm 0.1$ & $12.0 \pm 0.1$ & $\mathrm{U}$ & \\
\hline \multirow[t]{2}{*}{ TYC 7362-0724-1 } & $2010-06-16$ & $57(+211)$ & & & & & Electronic table \\
\hline & 2011-05-11 & 57 & & & & $\mathrm{~B}(+\mathrm{U})$ & Electronic table \\
\hline \multirow[t]{5}{*}{ TYC 8728-2262-1 } & $2011-08-25$ & cc-1 & $2821 \pm 12$ & $254.8 \pm 0.3$ & $9.1 \pm 0.1$ & $\mathrm{U}$ & \\
\hline & $2011-08-25$ & cc-2 & $4449 \pm 13$ & $27.5 \pm 0.2$ & $11.4 \pm 0.1$ & $\mathrm{U}$ & \\
\hline & $2011-08-25$ & cc-3 & $6232 \pm 14$ & $130.2 \pm 0.1$ & $12.1 \pm 0.2$ & $\mathrm{U}$ & \\
\hline & 2011-08-25 & cc-4 & $6399 \pm 12$ & $99.3 \pm 0.2$ & $6.3 \pm 0.0$ & $\mathrm{U}$ & \\
\hline & $2011-08-25$ & cc-5 & $6883 \pm 12$ & $166.3 \pm 0.1$ & $12.4 \pm 0.1$ & $\mathrm{U}$ & \\
\hline \multirow[t]{3}{*}{ HIP 86672} & $2010-06-16$ & 261 & & & & & Electronic table \\
\hline & 2011-08-25 & none & & & & & \\
\hline & $2013-04-25$ & $80(+181)$ & & & & $\mathrm{B}(+\mathrm{U})$ & Electronic table \\
\hline \multirow{2}{*}{ HIP 89829} & 2011-06-13 & 99 & & & & & Electronic table \\
\hline & 2012-08-09 & $29(+70)$ & & & & $\mathrm{B}(+\mathrm{U})$ & Electronic table \\
\hline \multirow[t]{25}{*}{ HIP 93375} & 2010-06-14 & cc-1 & $3208 \pm 11$ & $121.6 \pm 0.2$ & $13.8 \pm 0.2$ & $\mathrm{~B}$ & \\
\hline & 2011-05-30 & cc-1 & $3175 \pm 9$ & $120.5 \pm 0.2$ & $13.6 \pm 0.2$ & B & \\
\hline & $2010-06-14$ & cc-2 & $4261 \pm 10$ & $9.2 \pm 0.2$ & $13.7 \pm 0.2$ & $\mathrm{~B}$ & \\
\hline & 2011-05-30 & $\mathrm{cc}-2$ & $4335 \pm 8$ & $9.0 \pm 0.2$ & $13.4 \pm 0.2$ & B & \\
\hline & 2010-06-14 & cc-3 & $4595 \pm 10$ & $264.5 \pm 0.2$ & $12.5 \pm 0.1$ & B & \\
\hline & 2011-05-30 & cc-3 & $4591 \pm 8$ & $265.7 \pm 0.2$ & $12.8 \pm 0.1$ & $\mathrm{~B}$ & \\
\hline & $2010-06-14$ & cc-4 & $4822 \pm 12$ & $139.6 \pm 0.1$ & $11.4 \pm 0.1$ & B & \\
\hline & 2011-05-30 & cc-4 & $4754 \pm 10$ & $138.9 \pm 0.1$ & $11.5 \pm 0.1$ & B & \\
\hline & 2010-06-14 & cc-5 & $5308 \pm 10$ & $173.7 \pm 0.2$ & $13.7 \pm 0.2$ & $\mathrm{~B}$ & \\
\hline & 2011-05-30 & cc-5 & $5218 \pm 8$ & $173.6 \pm 0.1$ & $13.5 \pm 0.1$ & B & \\
\hline & 2010-06-14 & cc-6 & $5354 \pm 10$ & $176.9 \pm 0.2$ & $14.0 \pm 0.2$ & $\mathrm{~B}$ & \\
\hline & 2011-05-30 & cc-6 & $5283 \pm 8$ & $177.1 \pm 0.1$ & $14.0 \pm 0.2$ & B & \\
\hline & 2010-06-14 & $\mathrm{cc}-7$ & $6095 \pm 10$ & $274.5 \pm 0.1$ & $12.0 \pm 0.1$ & B & \\
\hline & 2011-05-30 & cc-7 & $6104 \pm 8$ & $275.2 \pm 0.1$ & $12.7 \pm 0.1$ & B & \\
\hline & 2010-06-14 & cc- 8 & $6848 \pm 10$ & $185.1 \pm 0.1$ & $12.4 \pm 0.1$ & B & \\
\hline & 2011-05-30 & cc- 8 & $6763 \pm 8$ & $184.9 \pm 0.1$ & $11.6 \pm 0.1$ & $\mathrm{~B}$ & \\
\hline & $2010-06-14$ & cc-9 & $6942 \pm 12$ & $153.9 \pm 0.1$ & $12.4 \pm 0.1$ & B & \\
\hline & 2011-05-30 & cc- 9 & $6856 \pm 10$ & $154.2 \pm 0.1$ & $11.6 \pm 0.1$ & $\mathrm{~B}$ & \\
\hline & 2010-06-14 & cc-10 & $7089 \pm 14$ & $46.6 \pm 0.1$ & $12.9 \pm 0.1$ & B & \\
\hline & 2011-05-30 & cc-10 & $7143 \pm 12$ & $45.8 \pm 0.1$ & $13.5 \pm 0.2$ & B & \\
\hline & 2010-06-14 & cc-11 & $7502 \pm 13$ & $116.6 \pm 0.1$ & $11.1 \pm 0.1$ & $\mathrm{U}$ & \\
\hline & 2010-06-14 & cc-12 & $7512 \pm 12$ & $115.1 \pm 0.1$ & $12.6 \pm 0.1$ & $\mathrm{U}$ & \\
\hline & 2011-05-30 & cc-13 & $4157 \pm 9$ & $237.0 \pm 0.1$ & $14.1 \pm 0.2$ & $\mathrm{U}$ & \\
\hline & 2011-05-30 & cc-14 & $5917 \pm 8$ & $262.2 \pm 0.1$ & $14.4 \pm 0.3$ & $\mathrm{U}$ & \\
\hline & 2011-05-30 & cc- 15 & $9234 \pm 14$ & $322.3 \pm 0.1$ & $11.5 \pm 0.1$ & $\mathrm{U}$ & \\
\hline
\end{tabular}


G. Chauvin et al.: The VLT/NaCo large program to probe the occurrence of exoplanets in wide orbits. II.

Table 6. continued.

\begin{tabular}{|c|c|c|c|c|c|c|c|}
\hline Name-1 & UT-Date & Nb Cand. & $\begin{array}{l}\text { Sep } \\
\text { (mas) }\end{array}$ & $\begin{array}{l}\text { PA } \\
\text { (deg) }\end{array}$ & $\begin{array}{l}\Delta H \\
(\mathrm{mag})\end{array}$ & Status & Comments \\
\hline HIP 94235 & $2010-07-30$ & none & & & & & New binary (see Table 6) \\
\hline \multirow{3}{*}{ TYC 6893-1391-1 } & 2011-06-08 & cc-1 & $3289 \pm 4$ & $229.6 \pm 0.1$ & $11.2 \pm 0.2$ & $\mathrm{U}$ & \\
\hline & 2011-06-08 & cc-2 & $3373 \pm 1$ & $256.6 \pm 0.1$ & $11.8 \pm 0.3$ & $\mathrm{U}$ & \\
\hline & 2011-06-08 & cc-3 & $5761 \pm 7$ & $224.5 \pm 0.1$ & $6.8 \pm 0.0$ & $\mathrm{U}$ & \\
\hline TYC 5206-0915-1 & $2010-07-30$ & none & & & & & \\
\hline \multirow[t]{2}{*}{ TYC 5736-0649-1 } & 2011-08-18 & cc-1 & $4360 \pm 6$ & $206.3 \pm 0.1$ & $9.8 \pm 0.1$ & $\mathrm{U}$ & \\
\hline & 2011-08-18 & cc-2 & $6130 \pm 8$ & $306.9 \pm 0.1$ & $10.6 \pm 0.1$ & $\mathrm{U}$ & \\
\hline HD 189285 & 2011-08-20 & cc-1 & $4519 \pm 4$ & $24.9 \pm 0.1$ & $9.5 \pm 0.1$ & $\mathrm{U}$ & \\
\hline HIP 98470 & $2010-06-15$ & none & & & & & \\
\hline \multirow[t]{4}{*}{ TYC 5164-567-1 } & 2011-07-29 & cc-1 & $2632 \pm 3$ & $207.5 \pm 0.1$ & $3.3 \pm 0.0$ & $\mathrm{U}$ & \\
\hline & 2011-07-29 & cc-2 & $4421 \pm 5$ & $56.8 \pm 0.1$ & $11.2 \pm 0.1$ & $\mathrm{U}$ & \\
\hline & 2011-07-29 & cc-3 & $5674 \pm 7$ & $229.3 \pm 0.1$ & $8.6 \pm 0.1$ & $\mathrm{U}$ & \\
\hline & 2011-07-29 & cc-4 & $7254 \pm 9$ & $139.8 \pm 0.1$ & $9.8 \pm 0.1$ & $\mathrm{U}$ & \\
\hline HIP 99273 & $2010-07-31$ & none & & & & & \\
\hline HD 199058 & $2010-06-15$ & none & & & & & New binary (see Table 6) \\
\hline \multirow[t]{2}{*}{ HIP 105384} & $2010-07-31$ & none & & & & & \\
\hline & 2011-06-08 & cc-1 & $7038 \pm 7$ & $24.5 \pm 0.1$ & $14.1 \pm 0.2$ & $\mathrm{U}$ & \\
\hline HIP 105612 & 2010-07-31 & none & & & & & \\
\hline HIP 107684 & $2010-06-15$ & none & & & & & New binary (see Table 6 ) \\
\hline HIP 108422 & $2010-07-30$ & none & & & & & Known binary ${ }^{d}$ \\
\hline TYC 8004-0083-1 & $2010-06-15$ & none & & & & & \\
\hline HIP 114046 & $2010-06-15$ & none & & & & & \\
\hline \multirow[t]{2}{*}{ TYC 9338-2016-1 } & 2009-11-23 & none & & & & & \\
\hline & $2010-07-30$ & none & & & & & \\
\hline TYC 9529-0340-1 & 2010-07-31 & none & & & & & \\
\hline TYC 9339-2158-1 & $2010-07-31$ & none & & & & & \\
\hline TYC 6406-0180-1 & 2010-07-30 & none & & & & & \\
\hline HIP 116910 & 2009-11-22 & none & & & & & \\
\hline
\end{tabular}

